TAIWANESE JOURNAL OF MATHEMATICS

Vol. 19, No. 1, pp. 279-314, February 2015

DOI: $10.11650 /$ tjm.19.2015.4692

This paper is available online at http://journal.taiwanmathsoc.org.tw

\title{
LITTLEWOOD-PALEY CHARACTERIZATIONS OF ANISOTROPIC HARDY SPACES OF MUSIELAK-ORLICZ TYPE
}

\author{
Baode Li, Xingya Fan and Dachun Yang*
}

\begin{abstract}
Let $\varphi: \mathbb{R}^{n} \times[0, \infty) \rightarrow[0, \infty)$ be a Musielak-Orlicz function and $A$ an expansive dilation. Let $H_{A}^{\varphi}\left(\mathbb{R}^{n}\right)$ be the anisotropic Hardy space of MusielakOrlicz type defined via the grand maximal function. Its atomic characterization and some other maximal function characterizations of $H_{A}^{\varphi}\left(\mathbb{R}^{n}\right)$, in terms of the radial, the non-tangential and the tangential maximal functions, are known. In this article, the authors further obtain their characterizations in terms of the Lusin-area function, the $g$-function or the $g_{\lambda}^{*}$-function via first establishing an anisotropic Peetre's inequality of Musielak-Orlicz type. Moreover, the range of $\lambda$ in the $g_{\lambda}^{*}$ function characterization of $H_{A}^{\varphi}\left(\mathbb{R}^{n}\right)$ coincides with the known best conclusions in the case when $H_{A}^{\varphi}\left(\mathbb{R}^{n}\right)$ is the classical Hardy space $H^{p}\left(\mathbb{R}^{n}\right)$ or the anisotropic Hardy space $H_{A}^{p}\left(\mathbb{R}^{n}\right)$ or their weighted variants, where $p \in(0,1]$.
\end{abstract}

\section{INTRODUCTION}

Many fields in analysis require the study of specific function spaces. In harmonic analysis, one soon encounters the Lebesgue spaces, the Hardy spaces, various forms of the Lipschitz spaces, the space BMO and the Sobolev spaces. From the original definitions of these spaces, it may not appear that they are very closely related. There exist, however, various unified approaches to their study, the Littlewood-Paley theory, which arises naturally from the consideration of the Dirichlet problem, provides one of the most successful unifying perspectives on these function spaces (see [18] for

Received April 17, 2014, accepted June 11, 2014.

Communicated by Der-Chen Chang.

2010 Mathematics Subject Classification: Primary 42B25; Secondary 42B30, 42B35, 46E30.

Key words and phrases: Littlewood-Paley function, Anisotropic expansive dilation, Muckenhoupt weight, Musielak-Orlicz function, Hardy space.

This project is partially supported by the National Natural Science Foundation of China (Grant Nos. 11161044, 11171027 and 11361020).

Dachun Yang is also partially supported by the Specialized Research Fund for the Doctoral Program of Higher Education of China (Grant No. 20120003110003) and the Fundamental Research Funds for Central Universities of China (Grant Nos. 2012LYB26 and 2012CXQT09).

*Corresponding author. 
more details). Recall that the classical Hardy spaces defined via the grand maximal function can also be equivalently characterized via the Littlewood-Paley $g$-function, the Lusin-area function or the $g_{\lambda}^{*}$-function (see, for example, [23, 44]).

On the other hand, as a generalization of $L^{p}\left(\mathbb{R}^{n}\right)$, the Orlicz space was introduced by Birnbaum and Orlicz in [3] and Orlicz in [40]. Since then, the theory of the Orlicztype spaces themselves has been well developed and these spaces have been widely used in many branches of analysis (see, for example, [2, 25, 36, 29, 39]). Moreover, as a development of the theory of Orlicz spaces, Orlicz-Hardy spaces and their dual spaces were studied by Strömberg [42] and Janson [27] on $\mathbb{R}^{n}$ and Orlicz-Hardy spaces associated with divergence form elliptic operators by Jiang and Yang [28]. A survey on the real-variable theory of Orlicz-type function spaces associated with operators is recently given in [12].

Let $\mathcal{A}_{q}\left(\mathbb{R}^{n}\right)$ with $q \in[1, \infty]$ denote the classical class of Muckenhoupt weights (see, for example, [21] or [23] for their definitions and properties) and $\varphi$ be a growth function (see [30]) which means that $\varphi: \mathbb{R}^{n} \times[0, \infty) \rightarrow[0, \infty)$ is a Musielak-Orlicz function such that $\varphi(x, \cdot)$ is an Orlicz function uniformly in $x \in \mathbb{R}^{n}$ and $\varphi(\cdot, t)$ is a Muckenhoupt $\mathcal{A}_{\infty}\left(\mathbb{R}^{n}\right)$ weight uniformly in $t \in(0, \infty)$. It is known that Musielak-Orlicz functions naturally generalize Orlicz functions that may vary in the spatial variables (see, for example, [38, 30, 34, 15]). Recently, Ky [30] introduced a Musielak-Orlicz Hardy space $H^{\varphi}\left(\mathbb{R}^{n}\right)$, via the grand maximal function, and established its atomic characterization. Moreover, Liang and Yang [35], Hou et al. [24] and Liang et al. [34] further gave out the dual spaces and some other real-variable characterizations of $H^{\varphi}\left(\mathbb{R}^{n}\right)$ in terms of various maximal functions, the Littlewood-Paley $g$-function, the Lusin-area function or the $g_{\lambda}^{*}$-function.

Let $A$ be an expansive dilation on $\mathbb{R}^{n}$. Very recently, Li et al. [33] introduced the anisotropic Hardy space $H_{A}^{\varphi}\left(\mathbb{R}^{n}\right)$ of Musielak-Orlicz type via the anisotropic grand maximal function and established its anisotropic atomic characterization. These Hardy spaces include the classical Hardy spaces of Fefferman-Stein [16], the weighted anisotropic Hardy spaces of Bownik [8], and the Hardy spaces of Musielak-Orlicz type of Ky [30], as special cases. Motivated by [34] and [33], in this article, we further establish some new real-variable characterizations of $H_{A}^{\varphi}\left(\mathbb{R}^{n}\right)$ in terms of the Littlewood-Paley Lusin-area function, the $g$-function or the $g_{\lambda}^{*}$-function.

Precisely, this article is organized as follows.

In Section 2, we first recall the definitions of the anisotropic Hardy spaces of Musielak-Orlicz type via the grand maximal function (denoted by $H_{A}^{\varphi}\left(\mathbb{R}^{n}\right)$ ), their atomic variants (denoted by $H_{A}^{\varphi, q, s}\left(\mathbb{R}^{n}\right)$ ), and their variants via the Lusin-area function (denoted by $H_{S, A}^{\varphi}\left(\mathbb{R}^{n}\right)$ ). Then we show $H_{A}^{\varphi, q, s}\left(\mathbb{R}^{n}\right)=H_{S, A}^{\varphi}\left(\mathbb{R}^{n}\right)$ with equivalent (quasi-)norms by the anisotropic Calderon reproducing formula, which, together with the known conclusion $H_{A}^{\varphi, q, s}\left(\mathbb{R}^{n}\right)=H_{A}^{\varphi}\left(\mathbb{R}^{n}\right)$ (see [33, Theorem 40]), further implies that $H_{A}^{\varphi}\left(\mathbb{R}^{n}\right)=H_{S, A}^{\varphi}\left(\mathbb{R}^{n}\right)$ with equivalent (quasi-)norms. Here, we point out that, 
since we are working on anisotropic Hardy spaces of Musielak-Orlicz type, when we decompose a distribution into a sum of atoms, the classical dual method used for estimating the norm of each atom does not work anymore in the present setting. Instead, we invoke a more subtle estimate, involving rescaling techniques (see (2.22) below). Also, observe that the method used in this article is different from that used in [24] for the Lusin-area function characterization of $H^{\varphi}\left(\mathbb{R}^{n}\right)$, which heavily depends on the theory of tent spaces (mainly, the atomic characterizations of tent spaces) and the molecular characterization of $H^{\varphi}\left(\mathbb{R}^{n}\right)$. The method used in this article avoids tent spaces and the molecular characterization of $H_{A}^{\varphi}\left(\mathbb{R}^{n}\right)$ and, in the spirit, is more close to the classical one (see, for example, [13]).

In Section 3, motivated by [34, Theorems 4.4 and 4.8] and via borrowing some ideas from Folland and Stein [17] and Aguilera and Segovia [1], the anisotropic LittlewoodPaley $g$-function and the $g_{\lambda}^{*}$-function characterizations of $H_{A}^{\varphi}\left(\mathbb{R}^{n}\right)$ are established via the discrete anisotropic Calderon reproducing formula (see Lemma 3.2 below), an anisotropic Peetre's inequality of Musielak-Orlicz type (see Lemma 3.7 below) and a Musielak-Orlicz Fefferman-Stein vector-valued inequality (see Lemma 3.6 below). We point out that this method is different from that used by Liang et al. in the proof of [34, Theorem 4.4], in which a subtle pointwise upper estimate via the vector-valued HardyLittlewood maximal function (see [34, (4.2)]) was used. However, such a pointwise upper estimate is still unknown and we do not know whether it holds true or not in the present setting due to its anisotropic structure. Moreover, the range of $\lambda \in(2 q / p, \infty)$ in the anisotropic $g_{\lambda}^{*}$-function characterization of $H_{A}^{\varphi}\left(\mathbb{R}^{n}\right)$ (see Theorem 3.9 below) coincides with the known best conclusions in the case when $H_{A}^{\varphi}\left(\mathbb{R}^{n}\right)$ is the classical Hardy space $H^{p}\left(\mathbb{R}^{n}\right)$, the anisotropic Hardy space $H_{A}^{p}\left(\mathbb{R}^{n}\right)$ or their weighted variants, where $p \in(0,1]$ (see [34, p. 414] for more details and related references therein).

Finally, we make some conventions on notation. Let $\mathbb{N}:=\{1,2, \ldots\}$ and $\mathbb{Z}_{+}:=$ $\{0\} \cup \mathbb{N}$. Denote by $\mathcal{S}\left(\mathbb{R}^{n}\right)$ the space of all Schwartz functions and $\mathcal{S}^{\prime}\left(\mathbb{R}^{n}\right)$ its dual space (namely, the space of all tempered distributions). For any $\alpha:=\left(\alpha_{1}, \ldots, \alpha_{n}\right) \in \mathbb{Z}_{+}^{n}$, let $|\alpha|:=\alpha_{1}+\cdots+\alpha_{n}$ and $\partial^{\alpha}:=\left(\frac{\partial}{\partial x_{1}}\right)^{\alpha_{1}} \cdots\left(\frac{\partial}{\partial x_{n}}\right)^{\alpha_{n}}$. Throughout the whole paper, we denote by $C$ a positive constant which is independent of the main parameters, but it may vary from line to line. The symbol $D \lesssim F$ means that $D \leq C F$. If $D \lesssim F$ and $F \lesssim D$, we then write $D \sim F$. We also use $C_{(\alpha, \beta, \ldots)}$ to denote a positive constant depending on the parameters $\alpha, \beta, \ldots$. If $E$ is a subset of $\mathbb{R}^{n}$, we denote by $\chi_{E}$ its characteristic function. For any $a \in \mathbb{R},\lfloor a\rfloor$ denotes the maximal integer not larger than $a$.

\section{The Lusin-area Function Characterization of $H_{A}^{\varphi}\left(\mathbb{R}^{n}\right)$}

In this section, we first recall the notion of the anisotropic Hardy spaces of MusielakOrlicz type via the grand maximal function from [33], and then establish their characterization in terms of the anisotropic Lusin-area function. 
We begin with some notation on Orlicz functions; see, for example, [30]. A function $\phi:[0, \infty) \rightarrow[0, \infty)$ is called an Orlicz function, if it is nondecreasing and $\phi(0)=0, \phi(t)>0$ if $t>0$ and $\lim _{t \rightarrow \infty} \phi(t)=\infty$. Observe that, different from the classical Orlicz functions which are convex, the Orlicz functions in this article may not be convex. An Orlicz function $\phi$ is said to be of lower (resp., upper) type $p$ with $p \in(-\infty, \infty)$, if there exists a positive constant $C$ such that, for all $t \in[0, \infty)$ and $s \in(0,1)$ (resp., $s \in[1, \infty)$ ),

$$
\phi(s t) \leq C s^{p} \phi(t) .
$$

Given a function $\varphi: \mathbb{R}^{n} \times[0, \infty) \rightarrow[0, \infty)$ such that, for any $x \in \mathbb{R}^{n}, \varphi(x, \cdot)$ is an Orlicz function, $\varphi$ is said to be of uniformly lower (resp., upper) type $p$ with $p \in(-\infty, \infty)$, if there exists a positive constant $C$ such that, for all $x \in \mathbb{R}^{n}, t \in[0, \infty)$ and $s \in(0,1)$ (resp., $s \in[1, \infty)$ ),

$$
\varphi(x, s t) \leq C s^{p} \varphi(x, t) .
$$

Let

$$
i(\varphi):=\sup \{p \in(-\infty, \infty): \varphi \text { is of uniformly lower type } p\}
$$

denote the uniformly critical lower type of the function $\varphi$. Recall that $i(\varphi)$ may not be attainable; namely, $\varphi$ may not be of uniformly lower type $i(\varphi)$; see, for example, [34] for some examples.

Now we recall the notion of expansive dilations on $\mathbb{R}^{n}$; see [4]. A real $n \times n$ matrix $A$ is called an expansive dilation, shortly a dilation, if $\min _{\lambda \in \sigma(A)}|\lambda|>1$, where $\sigma(A)$ denotes the set of all eigenvalues of $A$. Let $\lambda_{-}$and $\lambda_{+}$be two positive numbers such that

$$
1<\lambda_{-}<\min \{|\lambda|: \lambda \in \sigma(A)\} \leq \max \{|\lambda|: \lambda \in \sigma(A)\}<\lambda_{+} .
$$

In the case when $A$ is diagonalizable over $\mathbb{C}$, we can even take

$$
\lambda_{-}:=\min \{|\lambda|: \lambda \in \sigma(A)\}
$$

and $\lambda_{+}:=\max \{|\lambda|: \lambda \in \sigma(A)\}$. Otherwise, we need to choose them sufficiently close to these equalities according to what we need in our arguments.

It was proved in [4, Lemma 2.2] that, for a given dilation $A$, there exist an open ellipsoid $\Delta$ and $r \in(1, \infty)$ such that $\Delta \subset r \Delta \subset A \Delta$, and one can and do additionally assume that $|\Delta|=1$, where $|\Delta|$ denotes the $n$-dimensional Lebesgue measure of the set $\Delta$. Let $B_{k}:=A^{k} \Delta$ for $k \in \mathbb{Z}$. Then $B_{k}$ is open, $B_{k} \subset r B_{k} \subset B_{k+1}$ and $\left|B_{k}\right|=b^{k}$, here and hereafter, $b:=|\operatorname{det} A|$. Throughout the whole paper, let $\sigma$ be the minimum integer such that $r^{\sigma} \geq 2$ and, for any subset $E$ of $\mathbb{R}^{n}$, let $E^{\complement}:=\mathbb{R}^{n} \backslash E$. Then, for all $k, j \in \mathbb{Z}$ with $k \leq j$, it holds true that

$$
\begin{aligned}
& B_{k}+B_{j} \subset B_{j+\sigma}, \\
& B_{k}+\left(B_{k+\sigma}\right)^{\complement} \subset\left(B_{k}\right)^{\complement},
\end{aligned}
$$


where $E+F$ denotes the algebraic sum $\{x+y: x \in E, y \in F\}$ of sets $E, F \subset \mathbb{R}^{n}$.

Definition 2.1. A quasi-norm, associated with an expansive matrix $A$, is a Borel measurable mapping $\rho_{A}: \mathbb{R}^{n} \rightarrow[0, \infty)$, for simplicity, denoted by $\rho$, satisfying

(i) $\rho(x)>0$ for all $x \in \mathbb{R}^{n} \backslash\left\{\overrightarrow{0}_{n}\right\}$, here and hereafter, $\left\{\overrightarrow{0}_{n}\right\}:=(0, \ldots, 0)$;

(ii) $\rho(A x)=b \rho(x)$ for all $x \in \mathbb{R}^{n}$, where, as above, $b:=|\operatorname{det} A|$;

(iii) $\rho(x+y) \leq H[\rho(x)+\rho(y)]$ for all $x, y \in \mathbb{R}^{n}$, where $H \in[1, \infty)$ is a constant independent of $x$ and $y$.

In the standard dyadic case $A:=2 I_{n \times n}, \rho(x):=|x|^{n}$ for all $x \in \mathbb{R}^{n}$ is an example of homogeneous quasi-norms associated with $A$, here and hereafter, $|\cdot|$ always denotes the Euclidean norm in $\mathbb{R}^{n}$ and $I_{n \times n}$ the $n \times n$ unit matrix.

It was proved, in [4, Lemma 2.4], that all homogeneous quasi-norms associated with a given dilation $A$ are equivalent. Therefore, for a given expansive dilation $A$, in what follows, for convenience, we always use the step homogeneous quasi-norm $\rho$ defined by setting, for all $x \in \mathbb{R}^{n}$,

$$
\rho(x):=\sum_{k \in \mathbb{Z}} b^{k} \chi_{B_{k+1} \backslash B_{k}}(x) \text { if } x \neq 0, \text { or else } \rho\left(\overrightarrow{0}_{n}\right):=0 .
$$

By (2.2) and (2.3), we know that, for all $x, y \in \mathbb{R}^{n}$,

$$
\rho(x+y) \leq b^{\sigma}(\max \{\rho(x), \rho(y)\}) \leq b^{\sigma}[\rho(x)+\rho(y)] ;
$$

see [4, p. 8]. Moreover, $\left(\mathbb{R}^{n}, \rho, d x\right)$ is a space of homogeneous type in the sense of Coifman and Weiss [14], where $d x$ denotes the n-dimensional Lebesgue measure.

Definition 2.2. Let $p \in[1, \infty)$. A function $\varphi: \mathbb{R}^{n} \times[0, \infty) \rightarrow[0, \infty)$ is said to satisfy the uniform anisotropic Muckenhoupt condition $\mathbb{A}_{p}(A)$, denoted by $\varphi \in \mathbb{A}_{p}(A)$, if there exists a positive constant $C$ such that, for all $t \in(0, \infty)$, when $p \in(1, \infty)$,

$$
\sup _{x \in \mathbb{R}^{n}} \sup _{k \in \mathbb{Z}}\left\{b^{-k} \int_{x+B_{k}} \varphi(y, t) d y\right\}\left\{b^{-k} \int_{x+B_{k}}[\varphi(y, t)]^{-\frac{1}{p-1}} d y\right\}^{p-1} \leq C
$$

and, when $p=1$,

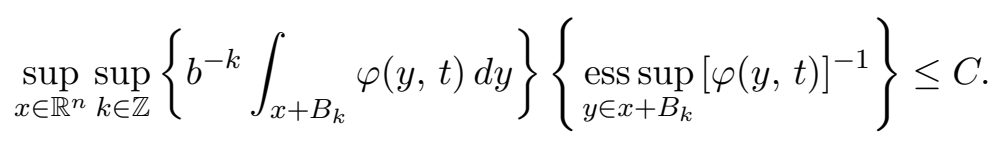

The minimal constant $C$ as above is denoted by $C_{(p, A, n, \varphi)}$.

Define $\mathbb{A}_{\infty}(A):=\bigcup_{1 \leq p<\infty} \mathbb{A}_{p}(A)$ and, for any $\varphi \in \mathbb{A}_{\infty}(A)$,

$$
q(\varphi):=\inf \left\{q \in[1, \infty): \varphi \in \mathbb{A}_{q}(A)\right\}
$$


If $\varphi \in \mathbb{A}_{\infty}(A)$ is independent of $t \in[0, \infty)$, then $\varphi$ is just an anisotropic Muckenhoupt $A_{\infty}(A)$ weight in [7]. Obviously, $q(\varphi) \in[1, \infty)$. Moreover, it is known (see [33]) that, if $q(\varphi) \in(1, \infty)$, then $\varphi \notin \mathbb{A}_{q(\varphi)}(A)$ and there exists a $\varphi \in\left(\cap_{q>1} \mathbb{A}_{q}(A)\right) \backslash \mathbb{A}_{1}(A)$ such that $q(\varphi)=1$.

Now, we recall the notion of anisotropic growth functions from [33].

Definition 2.3. A function $\varphi: \mathbb{R}^{n} \times[0, \infty) \rightarrow[0, \infty)$ is called an anisotropic growth function if

(i) The function $\varphi$ is a Musielak-Orlicz function, that is,

(a) the function $\varphi(x, \cdot):[0, \infty) \rightarrow[0, \infty)$ is an Orlicz function for all $x \in \mathbb{R}^{n}$,

(b) the function $\varphi(\cdot, t)$ is a Lebesgue measurable function for all $t \in[0, \infty)$;

(ii) the function $\varphi$ belongs to $\mathbb{A}_{\infty}(A)$;

(iii) the function $\varphi$ is of uniformly lower type $p$ for some $p \in(0,1]$ and of uniformly upper type 1 .

Given a growth function $\varphi$, let

$$
m(\varphi):=\left\lfloor\left[\frac{q(\varphi)}{i(\varphi)}-1\right] \frac{\ln b}{\ln \left(\lambda_{-}\right)}\right\rfloor,
$$

where $i(\varphi)$ and $q(\varphi)$ are, respectively, as in (2.1) and (2.4). Clearly,

$$
\varphi(x, t):=w(x) \Phi(t) \quad \text { for all } x \in \mathbb{R}^{n} \text { and } t \in[0, \infty)
$$

is an anisotropic growth function if $w$ is a classical or an anisotropic $A_{\infty}$ Muckenhoupt weight (see, for example, [7]) and $\Phi$ of lower type $p$ for some $p \in(0,1]$ and of upper type 1. More examples of growth functions can be found in [31, 32, 30, 34].

Remark 2.4. By [33, Lemma 11] (see also [30, Lemma 4.1]), without loss of generality, we may always assume that an anisotropic growth function $\varphi$ is of uniformly lower type $p$ for some $p \in(0,1]$ and of uniformly upper type 1 such that $\varphi(x, \cdot)$ is continuous and strictly increasing for all given $x \in \mathbb{R}^{n}$.

Recall that the Musielak-Orlicz-type space $L^{\varphi}\left(\mathbb{R}^{n}\right)$ is defined as the set of all measurable functions $f$ such that, for some $\lambda \in(0, \infty)$,

$$
\int_{\mathbb{R}^{n}} \varphi(x,|f(x)| / \lambda) d x<\infty
$$

with the Luxembourg (or called the Luxembourg-Nakano) (quasi-)norm

$$
\|f\|_{L^{\varphi}\left(\mathbb{R}^{n}\right)}:=\inf \left\{\lambda \in(0, \infty): \int_{\mathbb{R}^{n}} \varphi(x,|f(x)| / \lambda) d x \leq 1\right\} .
$$


In what follows, we denote by $\mathcal{S}\left(\mathbb{R}^{n}\right)$ the set of all Schwartz functions on $\mathbb{R}^{n}$ and by $\mathcal{S}^{\prime}\left(\mathbb{R}^{n}\right)$ its topological dual space. For $m \in \mathbb{N}$, let

$$
\mathcal{S}_{m}\left(\mathbb{R}^{n}\right):=\left\{\phi \in \mathcal{S}\left(\mathbb{R}^{n}\right): \sup _{x \in \mathbb{R}^{n},|\alpha| \leq m+1}[1+\rho(x)]^{m+2}\left|\partial^{\alpha} \phi(x)\right| \leq 1\right\} .
$$

In what follows, for $\phi \in \mathcal{S}\left(\mathbb{R}^{n}\right), k \in \mathbb{Z}$ and $x \in \mathbb{R}^{n}$, let $\phi_{k}(x):=b^{k} \phi\left(A^{k} x\right)$.

For $f \in \mathcal{S}^{\prime}\left(\mathbb{R}^{n}\right)$, the non-tangential grand maximal function $f_{m}^{*}$ of $f$ is defined by setting, for all $x \in \mathbb{R}^{n}$,

$$
f_{m}^{*}(x):=\sup _{\phi \in \mathcal{S}_{m}\left(\mathbb{R}^{n}\right)} \sup _{k \in \mathbb{Z}, y \in x+B_{k}}\left|f * \phi_{k}(y)\right| .
$$

If $m:=m(\varphi)$, we then write $f^{*}$ instead of $f_{m}^{*}$. The following anisotropic Hardy spaces $H_{m, A}^{\varphi}\left(\mathbb{R}^{n}\right)$ of Musielak-Orlicz type were introduced by Li et al. in [33].

Definition 2.5. For any $m \in \mathbb{N}$ and anisotropic growth function $\varphi$, the anisotropic Hardy space $H_{m, A}^{\varphi}\left(\mathbb{R}^{n}\right)$ of Musielak-Orlicz type is defined as the set of all $f \in \mathcal{S}^{\prime}\left(\mathbb{R}^{n}\right)$ such that $f_{m}^{*} \in L^{\varphi}\left(\mathbb{R}^{n}\right)$ with the (quasi-)norm $\|f\|_{H_{m, A}^{\varphi}\left(\mathbb{R}^{n}\right)}:=\left\|f_{m}^{*}\right\|_{L^{\varphi}\left(\mathbb{R}^{n}\right)}$. When $m:=m(\varphi), H_{m, A}^{\varphi}\left(\mathbb{R}^{n}\right)$ is denoted simply by $H_{A}^{\varphi}\left(\mathbb{R}^{n}\right)$.

Remark 2.6. By [33, Theorems 33, 40], we know that, if $m \geq m(\varphi)$, then the Musielak-Orlicz Hardy $H_{m, A}^{\varphi}\left(\mathbb{R}^{n}\right)$ is independent of $m$; see Lemma 2.13 below.

Observe that, when $A:=2 I_{n \times n}$ and $\varphi$ is as in (2.6) with a Muckenhoupt weight $w$ and an Orlicz function $\Phi$, the above Hardy spaces $H_{A}^{\varphi}\left(\mathbb{R}^{n}\right)$ are just weighted HardyOrlicz spaces which include classical Hardy-Orlicz spaces of Janson [26] ( $w \equiv 1$ in this context) and classical weighted Hardy spaces of García-Cuerva [19] as well as Strömberg and Torchinsky [43] $\left(\Phi(t):=t^{p}\right.$ for all $t \in[0, \infty)$ in this context); see also $[37,10,20]$. When $\varphi$ is as in (2.6) with $\Phi(t):=t^{p}$ for all $t \in[0, \infty)$, the above Hardy spaces $H_{A}^{\varphi}\left(\mathbb{R}^{n}\right)$ become weighted anisotropic Hardy spaces (see [8]).

Recall that a distribution $f \in \mathcal{S}^{\prime}\left(\mathbb{R}^{n}\right)$ is said to vanish weakly at infinity if, for any $\phi \in \mathcal{S}\left(\mathbb{R}^{n}\right), f * \phi_{k} \rightarrow 0$ in $\mathcal{S}^{\prime}\left(\mathbb{R}^{n}\right)$ as $k \rightarrow-\infty$. Denote by $\mathcal{S}_{0}^{\prime}\left(\mathbb{R}^{n}\right)$ the set of all $f \in \mathcal{S}^{\prime}\left(\mathbb{R}^{n}\right)$ vanishing weakly at infinity.

Definition 2.7. Let $\psi \in \mathcal{S}\left(\mathbb{R}^{n}\right)$ such that, for all multi-indices $\alpha$ satisfying $|\alpha| \leq$ $m(\varphi), \int_{\mathbb{R}^{n}} \psi(x) x^{\alpha} d x=0$, where $m(\varphi)$ is as in (2.5). For $f \in \mathcal{S}^{\prime}\left(\mathbb{R}^{n}\right)$ and $\lambda \in$ $(0, \infty)$, the Littlewood-Paley anisotropic Lusin-area function $S(f)$, the $g$-function $g(f)$ and the $g_{\lambda}^{*}$ function $g_{\lambda}^{*}(f)$ of $f$, associated to $\psi$, are defined, respectively, by setting, for all $x \in \mathbb{R}^{n}$,

$$
S(f)(x):=\left\{\sum_{k \in \mathbb{Z}} b^{-k} \int_{x+B_{k}}\left|f * \psi_{-k}(y)\right|^{2} d y\right\}^{1 / 2},
$$




$$
g(f)(x):=\left\{\sum_{k \in \mathbb{Z}}\left|\psi_{k} * f(x)\right|^{2}\right\}^{1 / 2}
$$

and

$$
g_{\lambda}^{*}(f)(x):=\left\{\sum_{k \in \mathbb{Z}} b^{-k} \int_{\mathbb{R}^{n}}\left[\frac{b^{k}}{b^{k}+\rho(x-y)}\right]^{\lambda}\left|f * \psi_{-k}(y)\right|^{2} d y\right\}^{1 / 2} .
$$

The anisotropic Musielak-Orlicz Hardy space $H_{S, A}^{\varphi}\left(\mathbb{R}^{n}\right)$ is defined as the set of all $f \in \mathcal{S}_{0}^{\prime}\left(\mathbb{R}^{n}\right)$ such that

$$
\|f\|_{H_{S, A}^{\varphi}\left(\mathbb{R}^{n}\right)}:=\|S(f)\|_{L^{\varphi}\left(\mathbb{R}^{n}\right)}<\infty .
$$

Similarly, the anisotropic Musielak-Orlicz Hardy space $H_{g, A}^{\varphi}\left(\mathbb{R}^{n}\right)$ or $H_{g_{\lambda}^{*}, A}^{\varphi}\left(\mathbb{R}^{n}\right)$ can also be defined with $S(f)$ replaced, respectively, by $g(f)$ or $g_{\lambda}^{*}(f)$.

The main result of this section is the following theorem.

Theorem 2.8. Let $\varphi$ be an anisotropic growth function as in Definition 2.3. Then

$$
H_{S, A}^{\varphi}\left(\mathbb{R}^{n}\right)=H_{A}^{\varphi}\left(\mathbb{R}^{n}\right)
$$

with equivalent (quasi-)norms.

To show Theorem 2.8, we need the atomic characterization of $H_{A}^{\varphi}\left(\mathbb{R}^{n}\right)$ from [33]. Let us begin with the following lemma from [9, Lemma 2.3], which is a slight modification of [11, Theorem 11].

Lemma 2.9. Let $A$ be a dilation. Then there exists a collection

$$
\mathcal{Q}:=\left\{Q_{\alpha}^{k} \subset \mathbb{R}^{n}: k \in \mathbb{Z}, \alpha \in \mathrm{I}_{k}\right\}
$$

of open subsets, where $\mathrm{I}_{k}$ is some index set, such that

(i) $\left|\mathbb{R}^{n} \backslash \cup_{\alpha} Q_{\alpha}^{k}\right|=0$ for each fixed $k$ and $Q_{\alpha}^{k} \cap Q_{\beta}^{k}=\emptyset$ if $\alpha \neq \beta$;

(ii) for all $\alpha, \beta, k, \ell$ with $\ell \geq k$, either $Q_{\alpha}^{k} \cap Q_{\beta}^{\ell}=\emptyset$ or $Q_{\alpha}^{\ell} \subset Q_{\beta}^{k}$;

(iii) for each $(\ell, \beta)$ and each $k<\ell$, there exists a unique $\alpha$ such that $Q_{\beta}^{\ell} \subset Q_{\alpha}^{k}$;

(iv) there exist some negative integer $v$ and positive integer $u$ such that, for all $Q_{\alpha}^{k}$ with $k \in \mathbb{Z}$ and $\alpha \in \mathrm{I}_{k}$, there exists $x_{Q_{\alpha}^{k}} \in Q_{\alpha}^{k}$ satisfying that, for all $x \in Q_{\alpha}^{k}$,

$$
x_{Q_{\alpha}^{k}}+B_{v k-u} \subset Q_{\alpha}^{k} \subset x+B_{v k+u} .
$$

In what follows, for convenience, we call $k$ the level of the dyadic cube $Q_{\alpha}^{k}$ with $k \in \mathbb{Z}$ and $\alpha \in \mathrm{I}_{k}$ and denote it by $\ell\left(Q_{\alpha}^{k}\right)$, and call $\mathcal{Q}$ of Lemma 2.9 dyadic cubes.

For any set $E \subset \mathbb{R}^{n}$ and $t \in(0, \infty)$, let $\varphi(E, t):=\int_{E} \varphi(x, t) d x$. 
Definition 2.10. For any $Q \in \mathcal{Q}$ and $q \in[1, \infty]$, let $L_{\varphi}^{q}(Q)$ be the set of all measurable functions $f$ supported in $Q$ such that

$$
\|f\|_{L_{\varphi}^{q}(Q)}:= \begin{cases}\sup _{t \in(0, \infty)}\left[\frac{1}{\varphi(Q, t)} \int_{\mathbb{R}^{n}}|f(x)|^{q} \varphi(x, t) d x\right]^{\frac{1}{q}}<\infty, & q \in[1, \infty), \\ \|f\|_{L^{\infty}(Q)}<\infty & q=\infty .\end{cases}
$$

It is easy to show that $\left(L_{\varphi}^{q}(Q),\|\cdot\|_{L_{\varphi}^{q}(Q)}\right)$ is a Banach space. Next we recall anisotropic atomic Hardy spaces of Musielak-Orlicz type from [33].

Definition 2.11. Let $\varphi$ be an anisotropic growth function and $q(\varphi)$ as in (2.4).

(i) An anisotropic triplet $(\varphi, q, s)$ is said to be admissible, if $q \in(q(\varphi), \infty]$ and $s \in \mathbb{Z}_{+}$such that $s \geq m(\varphi)$ with $m(\varphi)$ as in (2.5).

(ii) For an admissible anisotropic triplet $(\varphi, q, s)$, a measurable function $a$ is called an anisotropic $(\varphi, q, s)$-atom if

(a) $a \in L_{\varphi}^{q}(Q)$ for some dyadic cube $Q \in \mathcal{Q}$;

(b) $\|a\|_{L_{\varphi}^{q}(Q)} \leq\left\|\chi_{Q}\right\|_{L^{\varphi}\left(\mathbb{R}^{n}\right)}^{-1}$

(c) $\int_{\mathbb{R}^{n}} a(x) x^{\alpha} d x=0$ for any $|\alpha| \leq s$.

(iii) For an admissible anisotropic triplet $(\varphi, q, s)$, the anisotropic atomic Hardy space of Musielak-Orlicz type, $H_{A}^{\varphi, q, s}\left(\mathbb{R}^{n}\right)$, is defined as the set of all distributions $f \in \mathcal{S}^{\prime}\left(\mathbb{R}^{n}\right)$ which can be represented as a sum of multiples of anisotropic $(\varphi, q, s)$-atoms, that is, $f=\sum_{j} a_{j}$ in $\mathcal{S}^{\prime}\left(\mathbb{R}^{n}\right)$, where $a_{j}$ for $j$ is a multiple of an anisotropic $(\varphi, q, s)$-atom supported in the dyadic cube $Q_{j}$, with the property

$$
\sum_{j} \varphi\left(Q_{j},\left\|a_{j}\right\|_{L_{\varphi}^{q}\left(Q_{j}\right)}\right)<\infty
$$

Define

$$
\Lambda_{q}\left(\left\{a_{j}\right\}\right):=\inf \left\{\lambda \in(0, \infty): \sum_{j} \varphi\left(Q_{j}, \frac{\left\|a_{j}\right\|_{L_{\varphi}^{q}\left(Q_{j}\right)}}{\lambda}\right) \leq 1\right\}
$$

and

$$
\|f\|_{H_{A}^{\varphi, q, s}\left(\mathbb{R}^{n}\right)}:=\inf \left\{\Lambda_{q}\left(\left\{a_{j}\right\}\right): f=\sum_{j} a_{j} \text { in } \mathcal{S}^{\prime}\left(\mathbb{R}^{n}\right)\right\},
$$

where the infimum is taken over all admissible decompositions of $f$ as above.

Remark 2.12. In the definition of $(\varphi, q, s)$-atoms, if we replace dyadic cubes by dilated balls $\mathcal{B}:=\left\{x+B_{k}: x \in \mathbb{R}^{n}, k \in \mathbb{Z}\right\}$, which coincides with [33, Definition 30], by Lemma 2.9(iv), we know that both anisotropic atomic Hardy spaces of Musielak-Orlicz type are the same in the sense of equivalent (quasi-)norms. 
Combining Remark 2.12 and [33, Theorems 33 and 40], we obtain the following conclusion.

Lemma 2.13. Let $(\varphi, q, s)$ be admissible as in Definition 2.11. If $m \geq m(\varphi)$, then

$$
H_{A}^{\varphi}\left(\mathbb{R}^{n}\right)=H_{A}^{\varphi, q, s}\left(\mathbb{R}^{n}\right)=H_{m, A}^{\varphi}\left(\mathbb{R}^{n}\right)
$$

with equivalent (quasi-)norms.

Proof. This lemma is just a corollary of [33, Theorems 33 and 40]. Indeed, if $m \geq m(\varphi)$ and $(\varphi, q, s)$ is admissible, by [33, Theorem 33], we have

$$
H_{A}^{\varphi, q, s}\left(\mathbb{R}^{n}\right) \subset H_{A}^{\varphi, q, m(\varphi)}\left(\mathbb{R}^{n}\right) \subset H_{m, A}^{\varphi}\left(\mathbb{R}^{n}\right) .
$$

Conversely, if $m \geq m(\varphi),(\varphi, q, s)$ is admissible and $\widetilde{m}, \widetilde{s} \in \mathbb{Z}_{+}$such that

$$
\widetilde{m} \geq \widetilde{s} \geq \max \left\{m, s,\left\lfloor q(\varphi) \ln b /\left[i(\varphi) \ln \left(\lambda_{-}\right)\right]\right\rfloor\right\}
$$

with $i(\varphi)$ and $q(\varphi)$ being, respectively, as in (2.1) and (2.4), then, by Definition 2.7, [33, Theorem 40] and Definition 2.11, together with Remark 2.12, we find that

$$
H_{m, A}^{\varphi}\left(\mathbb{R}^{n}\right) \subset H_{\tilde{m}, A}^{\varphi}\left(\mathbb{R}^{n}\right) \subset H_{A}^{\varphi, q, \widetilde{s}}\left(\mathbb{R}^{n}\right) \subset H_{A}^{\varphi, q, s}\left(\mathbb{R}^{n}\right),
$$

which, combined with (2.9), implies that the conclusions of Lemma 2.13 and hence completes its proof.

Then Theorem 2.8 is a direct consequence of Lemma 2.13 and the following theorem.

Theorem 2.14. Let $(\varphi, q, s)$ be admissible as in Definition 2.11. Then

$$
H_{S, A}^{\varphi}\left(\mathbb{R}^{n}\right)=H_{A}^{\varphi, q, s}\left(\mathbb{R}^{n}\right)
$$

with equivalent (quasi-)norms.

To show Theorem 2.14, we need several technical lemmas. The following conclusion comes from [43, pp. 7-8].

Lemma 2.15. Let $q \in[1, \infty)$ and $\varphi \in \mathbb{A}_{q}(A)$. Then there exists a positive constant $C$ such that, for all $k \in \mathbb{Z}, t \in(0, \infty), x \in \mathbb{R}^{n}$ and $E \subset x+B_{k}$,

$$
\frac{\varphi\left(x+B_{k}, t\right)}{\varphi(E, t)} \leq C \frac{\left|x+B_{k}\right|^{q}}{|E|^{q}} .
$$

The following lemma shows that anisotropic $(\varphi, q, s)$-atoms of Musielak-Orlicz type are in $H_{S, A}^{\varphi}\left(\mathbb{R}^{n}\right)$. 
Lemma 2.16. Let $(\varphi, q, s)$ be admissible as in Definition 2.11. Then there exists a positive constant $C:=C_{(\varphi, q, s)}$ such that, for any anisotropic $(\varphi, q, s)$-atom a associated with some dyadic cube $Q$,

$$
\int_{\mathbb{R}^{n}} \varphi(x, S(a)(x)) d x \leq C \varphi\left(Q,\|a\|_{L_{\varphi}^{q}(Q)}\right) .
$$

Proof. We just consider $q \in(q(\varphi), \infty)$ with $q(\varphi)$ as in (2.4). The case $q=\infty$ is easier, the details being omitted. Now let us write

$$
\begin{aligned}
\int_{\mathbb{R}^{n}} \varphi(x, S(a)(x)) d x & =\int_{x_{Q}+B_{v \ell(Q)+u+2 \sigma}} \varphi(x, S(a)(x)) d x+\int_{x_{Q}+\left(B_{v \ell(Q)+u+2 \sigma}\right)^{\mathrm{C}}} \cdots \\
& =: \mathrm{I}+\mathrm{II} .
\end{aligned}
$$

By Lemma 2.15 with $\varphi \in \mathbb{A}_{q}(A)$ and Lemma 2.9(iv), we conclude that, for any $t \in(0, \infty)$,

$$
\varphi\left(x_{Q}+B_{v \ell(Q)+u+2 \sigma}, t\right) \lesssim b^{2 q(u+\sigma)} \varphi\left(x_{Q}+B_{v \ell(Q)-u}, t\right) \lesssim \varphi(Q, t) .
$$

From this, Hölder's inequality and the boundedness in $L^{q}\left(\mathbb{R}^{n}, \varphi(\cdot, t)\right)$, uniformly in $t \in(0, \infty)$, of the Lusin-area function $S$ with $\varphi \in \mathbb{A}_{q}(A)$ (see [9, Theorem 3.2]), it follows that

$$
\begin{aligned}
\mathrm{I} \lesssim & \int_{x_{Q}+B_{v \ell(Q)+u+2 \sigma}}\left[1+\frac{S(a)(x)}{\|a\|_{L_{\varphi}^{q}(Q)}}\right] \varphi\left(x,\|a\|_{L_{\varphi}^{q}(Q)}\right) d x \\
\lesssim & \varphi\left(Q,\|a\|_{L_{\varphi}^{q}(Q)}\right)+\frac{1}{\|a\|_{L_{\varphi}^{q}(Q)}}\left\{\int_{x_{Q}+B_{v \ell(Q)+u+2 \sigma}}[S(a)(x)]^{q} \varphi\left(x,\|a\|_{L_{\varphi}^{q}(Q)}\right) d x\right\}^{1 / q} \\
& \times\left[\varphi\left(x_{Q}+B_{v \ell(Q)+u+2 \sigma},\|a\|_{L_{\varphi}^{q}(Q)}\right)\right]^{1-1 / q} \lesssim \varphi\left(Q,\|a\|_{L_{\varphi}^{q}(Q)}\right) .
\end{aligned}
$$

To estimate II, we claim that, for all $j \in \mathbb{Z}_{+}$and

$$
\begin{gathered}
x \in U_{j}:=x_{Q}+\left(B_{v[\ell(Q)-j-1]+u+2 \sigma} \backslash B_{v[\ell(Q)-j]+u+2 \sigma}\right), \\
S(a)(x) \lesssim\|a\|_{L_{\varphi}^{q}(Q)}\left[b\left(\lambda_{-}\right)^{s+1}\right]^{v j}
\end{gathered}
$$

where $s \geq m(\varphi)$ with $m(\varphi)$ as in (2.5). Assume the claim holds true for the moment. Choose $\widetilde{q}>q(\varphi)$ and $p<i(\varphi)$ such that $b^{-\widetilde{q}+p}\left(\lambda_{-}\right)^{(s+1) p}>1$ (which is possible, since $s \geq m(\varphi)$ ), then, by the claim, $v<0$ as in Lemma 2.9(iv), the uniformly lower type $p$ 
of $\varphi$, Lemma 2.15 with $\varphi \in \mathbb{A}_{\widetilde{q}}(A)$ and Lemma 2.9(iv), we have

$$
\begin{aligned}
\mathrm{II} & \lesssim \sum_{j=1}^{\infty} \int_{U_{j}}\left[b\left(\lambda_{-}\right)^{s+1}\right]^{v j p} \varphi\left(x,\|a\|_{L_{\varphi}^{q}(Q)}\right) d x \\
& \lesssim \sum_{j=1}^{\infty}\left[b\left(\lambda_{-}\right)^{s+1}\right]^{v j p} \varphi\left(x_{Q}+B_{v[\ell(Q)-j-1]+u+2 \sigma},\|a\|_{L_{\varphi}^{q}(Q)}\right) \\
& \lesssim \varphi\left(Q,\|a\|_{L_{\varphi}^{q}(Q)}\right) \sum_{j=1}^{\infty}\left[b\left(\lambda_{-}\right)^{s+1}\right]^{v j p} b^{-v j \widetilde{q}} \lesssim \varphi\left(Q,\|a\|_{L_{\varphi}^{q}(Q)}\right) .
\end{aligned}
$$

Combining the estimates for I and II, we know that (2.10) holds true.

Thus, to finish the proof of (2.10), we still need to show the claim (2.11). To this end, for any $x \in U_{j}$, let

$$
[S(a)(x)]^{2}=\left[\sum_{k>-v \ell(Q)-u}+\sum_{k \leq-v \ell(Q)-u}\right] b^{k} \int_{y \in x+B_{-k}}\left|a * \phi_{k}(y)\right|^{2} d y=: \mathrm{II}_{1}+\mathrm{II}_{2} .
$$

To estimate $\mathrm{II}_{1}$, observe that, when $-k<v \ell(Q)+u$, since $\phi \in \mathcal{S}\left(\mathbb{R}^{n}\right)$, pick some $N \in \mathbb{N}$ to be fixed later, we conclude that, for all $y \in x+B_{-k}$,

$$
\begin{aligned}
\left|a * \phi_{k}(y)\right| & \lesssim b^{k} \int_{Q}\left|a(z) \phi\left(A^{k}(y-z)\right)\right| d z \\
& \lesssim b^{k} \int_{Q}|a(z)| \frac{1}{\left[1+\rho\left(A^{k}(y-z)\right)\right]^{N+1}} d z .
\end{aligned}
$$

Using $-k<v \ell(Q)+u$ and (2.3), we have

$$
y \in x+B_{-k} \subset x_{Q}+\left(B_{v[\ell(Q)-j]+u+2 \sigma}\right)^{\complement}+B_{-k} \subset x_{Q}+\left(B_{v[\ell(Q)-j]+u+\sigma}\right)^{\complement} .
$$

By this and $z \in x_{Q}+B_{v \ell(Q)+u}$, using (2.3) again, we obtain $y-z \in\left(B_{v[\ell(Q)-j]+u}\right)^{\complement}$, which implies that

$$
\rho(y-z) \geq b^{v[\ell(Q)-j]+u} .
$$

Moreover, by Hölder's inequality and

$$
\left[\int_{Q}|\varphi(z, t)|^{-\frac{q^{\prime}}{q}} d z\right]^{\frac{1}{q^{\prime}}} \lesssim \frac{b^{v \ell(Q)}}{[\varphi(Q, t)]^{\frac{1}{q}}}
$$

we see that, for all $y \in x+B_{-k}$,

$$
\begin{aligned}
\int_{Q}|a(z)| d z & \leq\left[\int_{Q}|a(z)|^{q} \varphi(z, t) d z\right]^{\frac{1}{q}}\left\{\int_{Q}[\varphi(z, t)]^{-\frac{q^{\prime}}{q}} d z\right\}^{\frac{1}{q^{\prime}}} \\
& \lesssim b^{v \ell(Q)}\|a\|_{L_{\varphi}^{q}(Q) .}
\end{aligned}
$$


Hence, by (2.12), (2.13) and (2.14), we find that, for all $y \in x+B_{-k}$,

$$
\left|a * \phi_{k}(y)\right| \lesssim b^{k-(N+1)\{k+v[\ell(Q)-j]+u\}+v \ell(Q)}\|a\|_{L_{\varphi}^{q}(Q)} \lesssim b^{-N\{k+v[\ell(Q)-j]\}}\|a\|_{L_{\varphi}^{q}(Q)} .
$$

Therefore, choosing $N \in \mathbb{N}$ such that $b^{N}>b\left(\lambda_{-}\right)^{s+1}$, we conclude that

$$
\begin{aligned}
\mathrm{II}_{1} & \lesssim \sum_{k>-v \ell(Q)-u} b^{-2 N\{k+v[\ell(Q)-j]\}}\|a\|_{L_{\varphi}^{q}(Q)}^{2} \\
& \lesssim b^{2 N j v}\|a\|_{L_{\varphi}^{q}(Q)}^{2} \lesssim\left[b\left(\lambda_{-}\right)^{s+1}\right]^{2 j v}\|a\|_{L_{\varphi}^{q}(Q)}^{2} .
\end{aligned}
$$

To estimate $\mathrm{II}_{2}$, since $-k \geq v \ell(Q)+u$ and $a$ has the vanishing moments up to order $s$, by Taylor's remainder theorem and $\phi \in \mathcal{S}\left(\mathbb{R}^{n}\right)$, we know that there exists some $\xi \in Q$ such that, for all $y \in x+B_{-k}$,

$$
\begin{aligned}
& \left|a * \phi_{k}(y)\right| \\
= & b^{k}\left|\int_{Q} a(z)\left\{\phi\left(A^{k}(y-z)\right)-\sum_{|\alpha| \leq s} \frac{D^{\alpha} \phi\left(A^{k}\left(y-x_{Q}\right)\right)}{\alpha !}\left[A^{k}\left(x_{Q}-z\right)\right]^{\alpha}\right\} d z\right| \\
\lesssim & b^{k} \int_{Q}|a(z)|\left|A^{k}\left(x_{Q}-z\right)\right|^{s+1} \sup _{\xi \in Q}\left[1+\rho\left(A^{k}(y-\xi)\right)\right]^{-N-1} d z .
\end{aligned}
$$

By using $A^{k}\left(z-x_{Q}\right) \in B_{k+v \ell(Q)+u}$, the fact $\left|A^{m} x\right| \lesssim\left(\lambda_{-}\right)^{m}$ for $m<0$ and $x \in B_{0}$ (see [8, (2.7)]), and $k+v \ell(Q)+u \leq 0$, we obtain

$$
\left|A^{k}\left(z-x_{Q}\right)\right| \lesssim\left(\lambda_{-}\right)^{k+v \ell(Q)+u} .
$$

Moreover, if $v[\ell(Q)-j]+u>-k$, by (2.3), we have

$$
y \in x+B_{-k} \subset x_{Q}+\left(B_{v[\ell(Q)-j]+u+2 \sigma}+B_{-k}\right)^{\complement} \subset x_{Q}+\left(B_{v[\ell(Q)-j]+u+\sigma}\right)^{\complement} .
$$

Using this, $\xi \in Q \subset x_{Q}+B_{v \ell(Q)+u}$, by (2.3) again, we further have $y-\xi \in$ $\left(B_{v[\ell(Q)-j]+u}\right)^{\complement}$, which implies that

$$
\rho\left(A^{k}(y-\xi)\right) \geq b^{k+v[\ell(Q)-j]+u}
$$

and hence

$$
\sup _{\xi \in Q}\left[1+\rho\left(A^{k}(y-\xi)\right)\right]^{-N-1} \leq \min \left\{1, b^{-N(k+v[\ell(Q)-j]+u)}\right\} .
$$

If $v[\ell(Q)-j]+u \leq-k$, since $b^{-N\{k+v[\ell(Q)-j]+u\}} \geq 1$, it is easy to see that (2.16) still holds true. 
Write

$$
\begin{aligned}
\mathrm{II}_{2} & =\left[\sum_{-v[\ell(Q)-j]-u<k \leq-v \ell(Q)-u}+\sum_{k \leq-v[\ell(Q)-j]-u}\right] b^{k} \int_{x+B_{-k}}\left|a * \phi_{k}(y)\right|^{2} d y \\
& =: \mathrm{II}_{2,1}+\mathrm{II}_{2,2} .
\end{aligned}
$$

Choosing $N \in \mathbb{N}$ such that $b^{N}>b\left(\lambda_{-}\right)^{s+1}$ and applying (2.14), (2.15) and (2.16), we obtain

$$
\begin{aligned}
\mathrm{II}_{2,1} & \lesssim \sum_{-v[\ell(Q)-j]-u<k \leq-v \ell(Q)-u}\left[\|a\|_{L_{\varphi}^{q}(Q)}\left(\lambda_{-}\right)^{(s+1)[k+v \ell(Q)+u]} b^{v \ell(Q)+k-N\{k+v[\ell(Q)-j]+u\}}\right]^{2} \\
& \lesssim\left[b\left(\lambda_{-}\right)^{s+1}\right]^{2 j v}\|a\|_{L_{\varphi}^{q}(Q)}^{2}
\end{aligned}
$$

and

$$
\begin{aligned}
\mathrm{II}_{2,2} & \lesssim \sum_{k \leq-v[\ell(Q)-j]-u}\left[\|a\|_{L_{\varphi}^{q}(Q)}\left(\lambda_{-}\right)^{(s+1)\{k+v \ell(Q)+u\}} b^{k+v \ell(Q)}\right]^{2} \\
& \lesssim\left[b\left(\lambda_{-}\right)^{s+1}\right]^{2 j v}\|a\|_{L_{\varphi}^{q}(Q)}^{2} .
\end{aligned}
$$

Combining the estimates of $\mathrm{II}_{1}, \mathrm{II}_{2,1}$ and $\mathrm{II}_{2,2}$, we know that (2.11) holds true, which completes the proof of Lemma 2.16 .

Lemma 2.17. Let $(\varphi, q, s)$ be admissible as in Definition 2.11. Then

$$
H_{A}^{\varphi, q, s}\left(\mathbb{R}^{n}\right) \subset \mathcal{S}_{0}^{\prime}\left(\mathbb{R}^{n}\right) .
$$

Proof. We show this lemma by borrowing some ideas from the proof of [24, Lemma 4.14].

For $m:=m(\varphi)$ with $m(\varphi)$ as in (2.5), $k \in \mathbb{Z}, \phi \in \mathcal{S}\left(\mathbb{R}^{n}\right), f \in H_{A}^{\varphi}\left(\mathbb{R}^{n}\right), x \in$ $\mathbb{R}^{n}$ and $y \in x+B_{k}$, we have $\left|f * \phi_{k}(x)\right| \leq\|\phi\|_{\mathcal{S}_{m}\left(\mathbb{R}^{n}\right)} f^{*}(y)$. Moreover, noticing that, for any $p \in(0, i(\varphi)), \varphi$ is of uniformly lower type $p$, then, by the uniformly lower type $p$ and the uniformly upper type 1 properties of $\varphi$ and the property that $\int_{\mathbb{R}^{n}} \varphi\left(x,|g(x)| /\|g\|_{L^{\varphi}\left(\mathbb{R}^{n}\right)}\right) d x=1$ for all $g \in L^{\varphi}\left(\mathbb{R}^{n}\right) \backslash\{0\}$ (see [30, Lemma 4.2(i)]), we conclude that, for all $x \in \mathbb{R}^{n}$,

$$
\begin{aligned}
& \min \left\{\left|f * \phi_{k}(x)\right|^{p},\left|f * \phi_{k}(x)\right|\right\} \\
& \lesssim\|\phi\|_{\mathcal{S}_{m}\left(\mathbb{R}^{n}\right)}\left[\varphi\left(x+B_{k}, 1\right)\right]^{-1} \int_{x+B_{k}} \varphi(y, 1) \min \left\{\left[f^{*}(y)\right]^{p}, f^{*}(y)\right\} d y \\
& \lesssim\|\phi\|_{\mathcal{S}_{m}\left(\mathbb{R}^{n}\right)}\left[\varphi\left(x+B_{k}, 1\right)\right]^{-1} \int_{x+B_{k}} \varphi\left(y, f^{*}(y)\right) d y \\
& \lesssim\|\phi\|_{\mathcal{S}_{m}\left(\mathbb{R}^{n}\right)}\left[\varphi\left(x+B_{k}, 1\right)\right]^{-1} \max \left\{\|f\|_{H_{A}^{\varphi}\left(\mathbb{R}^{n}\right)}^{p},\|f\|_{H_{A}^{\varphi}\left(\mathbb{R}^{n}\right)}\right\} \rightarrow 0,
\end{aligned}
$$


as $k \rightarrow-\infty$, which implies that $f$ vanishes weakly at infinity. Combining this and Lemma 2.13, we then complete the proof of Lemma 2.17.

Using Lemmas 2.16 and 2.17, with a slight modification on the proof of [30, Theorem 5.1], we have the following conclusion, the details being omitted.

Lemma 2.18. Let $(\varphi, q, s)$ be admissible as in Definition 2.11. Then $H_{A}^{\varphi, q, s}\left(\mathbb{R}^{n}\right) \subset$ $H_{S, A}^{\varphi}\left(\mathbb{R}^{n}\right)$ and the inclusion is continuous.

The following Calderón reproducing formula is just [9, Proposition 2.14]. In what follows, for any $\psi \in L^{1}\left(\mathbb{R}^{n}\right)$ and $\xi \in \mathbb{R}^{n}$, let $\widehat{\psi}(\xi):=\int_{\mathbb{R}^{n}} e^{-2 \pi i \xi x} \psi(x) d x$.

Lemma 2.19. Let $s \in \mathbb{Z}_{+}$and $A$ be a dilation on $\mathbb{R}^{n}$. Then there exist $\theta, \psi \in$ $\mathcal{S}\left(\mathbb{R}^{n}\right)$ such that

(i) $\operatorname{supp} \theta \subset B_{\rho}\left(\overrightarrow{0}_{n}, 1\right):=\left\{x \in \mathbb{R}^{n}: \rho(x)<1\right\}, \int_{\mathbb{R}^{n}} x^{\gamma} \theta(x) d x=0$ for all $\gamma \in$ $\left(\mathbb{Z}_{+}\right)^{n}$ with $|\gamma| \leq s$, and $\widehat{\theta}(\xi) \geq C>0$ for all $\xi \in\left\{x \in \mathbb{R}^{n}: a \leq \rho(x) \leq b\right\}$, where $0<a<b<1$ are constants;

(ii) supp $\widehat{\psi}$ is compact and bounded away from the origin;

(iii) $\sum_{j \in \mathbb{Z}} \widehat{\psi}\left(\left(A^{*}\right)^{j} \xi\right) \widehat{\theta}\left(\left(A^{*}\right)^{j} \xi\right)=1$ for all $\xi \in \mathbb{R}^{n} \backslash\left\{\overrightarrow{0}_{n}\right\}$, where $A^{*}$ denotes the transpose of $A$.

Then, for all $f \in \mathcal{S}_{0}^{\prime}\left(\mathbb{R}^{n}\right), f=\sum_{j \in \mathbb{Z}} f * \psi_{j} * \theta_{j}$ in $\mathcal{S}^{\prime}\left(\mathbb{R}^{n}\right)$.

The following lemma says that the space $L^{q}\left(\mathbb{R}^{n}, \varphi(\cdot, t)\right)$ with $t \in(0, \infty)$ can be characterized by the Lusin-area function and the Littlewood-Paley $g$-function, respectively. Moreover, the conclusions of Lemma 2.20 associated to the Lusin-area function are just [9, Theorem 3.2] in the one parameter case.

Lemma 2.20. Let $\varphi \in \mathbb{A}_{\infty}(A)$ with $q \in(q(\varphi), \infty), t \in(0, \infty)$ and $\theta, \psi$ be as in Lemma 2.19. Suppose $\phi:=\theta$ or $\psi$. Then $f \in L^{q}\left(\mathbb{R}^{n}, \varphi(\cdot, t)\right)$ if and only if $f \in \mathcal{S}_{0}^{\prime}\left(\mathbb{R}^{n}\right)$ and $S(f) \in L^{q}\left(\mathbb{R}^{n}, \varphi(\cdot, t)\right)$ if and only if $f \in \mathcal{S}_{0}^{\prime}\left(\mathbb{R}^{n}\right)$ and $g(f) \in$ $L^{q}\left(\mathbb{R}^{n}, \varphi(\cdot, t)\right)$, where $S(f)$ and $g(f)$ are defined, respectively, as in (2.7) and (2.8) via replacing $\psi$ therein by $\phi$. Moreover, for all $f \in L^{q}\left(\mathbb{R}^{n}, \varphi(\cdot, t)\right),\|f\|_{L^{q}\left(\mathbb{R}^{n}, \varphi(\cdot, t)\right)} \sim$ $\|S(f)\|_{L^{q}\left(\mathbb{R}^{n}, \varphi(\cdot, t)\right)} \sim\|g(f)\|_{L^{q}\left(\mathbb{R}^{n}, \varphi(\cdot, t)\right)}$ hold true uniformly for all $t \in(0, \infty)$, where the implicit positive constants are independent of $f$ and $t$.

By a slight and obvious modification on the proof of [9, Theorem 3.2], namely, replacing two parameters therein by one parameter here, we can show Lemma 2.20, the details being omitted.

Recall that the anisotropic Hardy-Littlewood maximal function $\mathcal{M}_{A}(f)$ of any locally integrable function $f$ is defined by setting, for all $x \in \mathbb{R}^{n}$,

$$
\mathcal{M}_{A} f(x):=\sup _{x \in B, B \in \mathcal{B}} \frac{1}{|B|} \int_{B}|f(y)| d y
$$


The following Lemma 2.21 is essentially [7, Theorem 2.5], by observing that the positive constant $\widetilde{C}$ in Lemma 2.21 bellow only depends on $n, p, q$ and the weight constant $C_{(p, A, n, \varphi)}$ which is uniform on $t \in(0, \infty)$, the detail being omitted.

Lemma 2.21. Let $\varphi \in \mathbb{A}_{p}(A), p \in(1, \infty)$ and $q \in(1, \infty]$. Then the anisotropic Hardy-Littlewood maximal operator $\mathcal{M}_{A}$ is bounded on $L^{p}\left(\mathbb{R}^{n}, \varphi(\cdot, t)\right)$ uniformly for $t \in(0, \infty)$ and, moreover, there exists a positive constant $\widetilde{C}$, depending only on $n, p, q$ and $C_{(p, A, n, \varphi)}$ in Definition 2.2, such that, for all $t \in(0, \infty)$ and $\left\{f_{j}\right\}_{j \in \mathbb{N}} \subset$ $L^{p}\left(\mathbb{R}^{n}, \varphi(\cdot, t)\right)$,

$$
\left\|\left\{\sum_{j \in \mathbb{N}}\left[\mathcal{M}_{A}\left(f_{j}\right)\right]^{q}\right\}^{1 / q}\right\|_{L^{p}\left(\mathbb{R}^{n}, \varphi(\cdot, t)\right)} \leq \widetilde{C}\left\|\left\{\sum_{j \in \mathbb{N}}\left|f_{j}\right|^{q}\right\}^{1 / q}\right\|_{L^{p}\left(\mathbb{R}^{n}, \varphi(\cdot, t)\right)} .
$$

Proof of Theorem 2.14. By Lemma 2.18, we only need to show $H_{S, A}^{\varphi}\left(\mathbb{R}^{n}\right) \subset$ $H_{A}^{\varphi, q, s}\left(\mathbb{R}^{n}\right)$, which is proved by the following five steps.

Step 1. Decompose $f$ via the Calderon reproducing formula.

For any $k \in \mathbb{Z}$ and $f \in H_{S, A}^{\varphi}\left(\mathbb{R}^{n}\right)$, let $\Omega_{k}:=\left\{x \in \mathbb{R}^{n}: S(f)(x)>2^{k}\right\}$ and

$$
\mathcal{R}_{k}:=\left\{Q \in \mathcal{Q}:\left|Q \cap \Omega_{k}\right|>|Q| / 2,\left|Q \cap \Omega_{k+1}\right| \leq|Q| / 2\right\} .
$$

Then, for each dyadic cube $Q \in \mathcal{Q}$, there exists a unique $k \in \mathbb{Z}$ such that $Q \in \mathcal{R}_{k}$. Let $\ell(Q), v, u$ be the same as in Lemma 2.9 corresponding to $A$. For any $Q \in \mathcal{Q}$, let

$$
\widetilde{Q}:=\left\{(y, m) \in \mathbb{R}^{n} \times \mathbb{R}: y \in Q, m \sim v \ell(Q)+u\right\},
$$

here and hereafter, $m \sim v \ell(Q)+u$ always means

$$
v \ell(Q)+u+\sigma \leq m<v[\ell(Q)-1]+u+\sigma,
$$

where $\sigma$ is as in (2.2) and (2.3). Observe that, in the above inequality, $v$ is negative. Obviously, $\{\widetilde{Q}\}_{Q \in \mathcal{Q}}$ are mutually disjoint and

$$
\mathbb{R}^{n} \times \mathbb{R}=\bigcup_{k \in \mathbb{Z}} \bigcup_{Q \in \mathcal{R}_{k}} \widetilde{Q}
$$

Let $\theta$ and $\psi$ be as in Lemma 2.19 and let each $\theta$ have the vanishing moments up to order $s$ with $s \geq m(\varphi)$.

For all $f \in H_{S, A}^{\varphi}\left(\mathbb{R}^{n}\right)$ and $x \in \mathbb{R}^{n}$, by Lemma 2.19 , together with the properties of $f \in \mathcal{S}^{\prime}\left(\mathbb{R}^{n}\right)$ (see [41, p. 23, Theorem 3.13] or [22, Theorem 2.3.20]), and (2.19), we know that

$$
\begin{aligned}
f(x) & =\sum_{k \in \mathbb{Z}} f * \psi_{-k} * \theta_{-k}(x)=\int_{\mathbb{R}^{n} \times \mathbb{R}} f * \psi_{-m}(y) \theta_{-m}(x-y) d y d \sigma(m) \\
& =\int_{\bigcup_{k \in \mathbb{Z}} \bigcup_{Q \in \mathcal{R}_{k}} \widetilde{Q}} f * \psi_{-m}(y) \theta_{-m}(x-y) d y d \sigma(m)=\sum_{k \in \mathbb{Z}} \sum_{Q \in \mathcal{R}_{k}} e_{Q}(x)
\end{aligned}
$$


hold true in $\mathcal{S}^{\prime}\left(\mathbb{R}^{n}\right)$, where

$$
e_{Q}(x):=\int_{\widetilde{Q}} f * \psi_{-m}(y) \theta_{-m}(x-y) d y d \sigma(m),
$$

and $\sigma(m)$ is the counting measure on $\mathbb{R}$.

Let $\left\{Q_{k}^{\ell}\right\}_{\ell}$ be the set of all maximal dyadic cubes in $\mathcal{R}_{k}$. For any $Q$ in $\mathcal{R}_{k}$, by Lemma 2.9(ii), there exists a unique maximal dyadic cube $Q_{k}^{\ell}$ such that $Q \subset Q_{k}^{\ell}$. Then we further have $f=\sum_{k \in \mathbb{Z}} \sum_{\ell} a_{k}^{\ell}$ in $\mathcal{S}^{\prime}\left(\mathbb{R}^{n}\right)$, where

$$
a_{k}^{\ell}:=\sum_{Q \subset Q_{k}^{\ell}, Q \in \mathcal{R}_{k}} e_{Q} .
$$

Step 2. Prove $\|f\|_{H_{A}^{\varphi, q, s}\left(\mathbb{R}^{n}\right)} \lesssim\|f\|_{H_{S, A}^{\varphi}\left(\mathbb{R}^{n}\right)}$.

First, we need a subtle estimate as follows.

Let $\mathcal{G}$ be any set of dyadic cubes in $\mathbb{R}^{n}$ and $e_{Q}$ as in (2.20) for all $Q \in \mathcal{G}$. Then we claim that, for all $x \in \mathbb{R}^{n}$,

$$
\left[S\left(\sum_{Q \in \mathcal{G}} e_{Q}\right)(x)\right]^{2} \lesssim \sum_{Q \in \mathcal{G}}\left[\mathcal{M}_{A}\left(c_{Q} \chi_{Q}\right)(x)\right]^{2},
$$

where

$$
c_{Q}:=\left\{\int_{\widetilde{Q}}\left|\psi_{-m} * f(y)\right|^{2} d y \frac{d \sigma(m)}{b^{m}}\right\}^{1 / 2} .
$$

Indeed, we can show the above claim by borrowing some ideas from [9, Lemma 4.7]. For any $Q \in \mathcal{Q}$, let $Q^{\prime}:=c_{Q}+B_{v[\ell(Q)-1]+u+2 \sigma}$. By repeating the proof of $[9$, Lemma 4.7, pp.412-413] via replacing two parameters therein by one parameter here, we conclude that

$$
\begin{aligned}
& {\left[S\left(\sum_{Q \in \mathcal{G}} e_{Q}\right)(x)\right]^{2}} \\
& \lesssim \sum_{Q \in \mathcal{Q}}\left(c_{Q}\right)^{2}\left[\mathcal{M}_{A}\left(\chi_{Q}\right)(x)\right]^{2}\left\{\sum_{P \in \mathcal{Q}, Q^{\prime} \cap P^{\prime} \neq \emptyset} b^{(s+1) v|\ell(Q)-\ell(P)| \log _{b}\left(\lambda_{-}\right)}\right\} .
\end{aligned}
$$

Combining this and the following estimate (see $[9,(4.18)]$ in one parameter case)

$$
\sum_{P \in \mathcal{Q}, Q^{\prime} \cap P^{\prime} \neq \emptyset} b^{(s+1) v|\ell(Q)-\ell(P)| \log _{b}\left(\lambda_{-}\right)} \lesssim 1,
$$

we obtain the desired claim. 
By Lemma 2.20, (2.22) and Lemma 2.21 with $\varphi \in \mathbb{A}_{q}(A)$, where $q \in(q(\varphi), \infty)$, we find that

$$
\begin{aligned}
& \left\|a_{k}^{\ell}\right\|_{L^{q}\left(\mathbb{R}^{n}, \varphi(\cdot, t)\right)} \\
& =\left\|\sum_{Q \subset Q_{k}^{\ell}, Q \in \mathcal{R}_{k}} e_{Q}\right\|_{L^{q}\left(\mathbb{R}^{n}, \varphi(\cdot, t)\right)} \lesssim\left\|S\left(\sum_{Q \subset Q_{k}^{\ell}, Q \in \mathcal{R}_{k}} e_{Q}\right)\right\|_{L^{q}\left(\mathbb{R}^{n}, \varphi(\cdot, t)\right)} \\
& \lesssim\left\|\left\{\sum_{Q \subset Q_{k}^{\ell}, Q \in \mathcal{R}_{k}}\left[\mathcal{M}_{A}\left(c_{Q} \chi_{Q}\right)\right]^{2}\right\}^{1 / 2}\right\|_{L^{q}\left(\mathbb{R}^{n}, \varphi(\cdot, t)\right)} \\
& \left.\lesssim \| \sum_{Q \subset Q_{k}^{\ell}, Q \in \mathcal{R}_{k}}\left(c_{Q}\right)^{2} \chi_{Q}\right]^{1 / 2} \|_{L^{q}\left(\mathbb{R}^{n}, \varphi(\cdot, t)\right)} .
\end{aligned}
$$

Notice that, for any $Q \in \mathcal{R}_{k}$ and $x \in Q$, by Lemma 2.9(iv), we conclude that

$$
\mathcal{M}_{A}\left(\chi_{\Omega_{k}}\right)(x) \geq \frac{1}{b^{v \ell(Q)+u}} \int_{x_{Q}+B_{v \ell(Q)+u}} \chi_{\Omega_{k}}(y) d y>b^{-2 u} \frac{\left|\Omega_{k} \cap Q\right|}{|Q|}>\frac{1}{2 b^{2 u}},
$$

which implies that

$$
\bigcup_{Q \subset Q_{k}^{\ell}, Q \in \mathcal{R}_{k}} Q \subset \widetilde{\Omega}_{k}:=\left\{x \in \mathbb{R}^{n}: \mathcal{M}_{A}\left(\chi_{\Omega_{k}}\right)(x)>\left(2 b^{2 u}\right)^{-1}\right\} .
$$

Moreover, for any $Q \in \mathcal{R}_{k}$ and $x \in Q$, by $Q \subset \widetilde{\Omega}_{k}$ and Lemma 2.9(iv), we have

$$
\mathcal{M}_{A}\left(\chi_{Q \cap\left(\widetilde{\Omega}_{k} \backslash \Omega_{k+1}\right)}\right)(x) \gtrsim \frac{1}{|Q|} \int_{Q} \chi_{\widetilde{\Omega}_{k} \backslash \Omega_{k+1}}(y) d y \gtrsim \frac{|Q|-|Q| / 2}{|Q|} \gtrsim \chi_{Q}(x) .
$$

From this and Lemma 2.21, it follows that

$$
\begin{aligned}
& \left\|a_{k}^{\ell}\right\|_{L^{q}\left(\mathbb{R}^{n}, \varphi(\cdot, t)\right)} \\
& \lesssim\left\|\left\{\sum_{Q \subset Q_{k}^{\ell}, Q \in \mathcal{R}_{k}}\left[\mathcal{M}_{A}\left(c_{Q} \chi_{Q \cap\left(\widetilde{\Omega}_{k} \backslash \Omega_{k+1}\right)}\right)\right]^{2}\right\}^{1 / 2}\right\|_{L^{q}\left(\mathbb{R}^{n}, \varphi(\cdot, t)\right)} \\
& \lesssim\left\|\left[\sum_{Q \subset Q_{k}^{\ell}, Q \in \mathcal{R}_{k}}\left(c_{Q}\right)^{2} \chi_{Q \cap\left(\widetilde{\Omega}_{k} \backslash \Omega_{k+1}\right)}\right]^{1 / 2}\right\|_{L^{q}\left(\mathbb{R}^{n}, \varphi(\cdot, t)\right)} .
\end{aligned}
$$


For any $Q \in \mathcal{R}_{k}, x \in Q$ and $(y, m) \in \widetilde{Q}$, by Lemma 2.9(iv) and (2.18), we conclude that $x-y \in B_{v \ell(Q)+u+\sigma} \subset B_{m}$, which, together with the disjointness of $\{\widetilde{Q}\}_{Q \subset Q_{k}^{\ell}}$, implies that

$$
\begin{aligned}
& \sum_{Q \subset Q_{k}^{\ell}, Q \in \mathcal{R}_{k}}\left(c_{Q}\right)^{2} \chi_{Q \cap\left(\widetilde{\Omega}_{k} \backslash \Omega_{k+1}\right)}(x) \\
= & \sum_{Q \subset Q_{k}^{\ell}, Q \in \mathcal{R}_{k}} \int_{\widetilde{Q}}\left|\psi_{-m} * f(y)\right|^{2} d y \frac{d \sigma(m)}{b^{m}} \chi_{Q \cap\left(\widetilde{\Omega}_{k} \backslash \Omega_{k+1}\right)}(x) \\
\lesssim & {[S(f)(x)]^{2} \chi_{Q_{k}^{\ell} \cap\left(\widetilde{\Omega}_{k} \backslash \Omega_{k+1}\right)}(x) . }
\end{aligned}
$$

Therefore, for any $t \in(0, \infty)$, by (2.24) and (2.25), we further have

$$
\begin{aligned}
\left\|a_{k}^{\ell}\right\|_{L^{q}\left(\mathbb{R}^{n}, \varphi(\cdot, t)\right)} & \lesssim\left\{\int_{\mathbb{R}^{n}}[S(f)(x)]^{q} \chi_{Q_{k}^{\ell} \cap\left(\widetilde{\Omega}_{k} \backslash \Omega_{k+1}\right)}(x) \varphi(x, t) d x\right\}^{1 / q} \\
& \lesssim 2^{k}\left[\varphi\left(Q_{k}^{\ell}, t\right)\right]^{\frac{1}{q}},
\end{aligned}
$$

which implies that $\left\|a_{k}^{\ell}\right\|_{L_{\varphi}^{q}\left(Q_{k}^{\ell}\right)} \leq \widetilde{C} 2^{k}$, where $\widetilde{C}$ is a positive constant independent of $k$ and $\ell$. By the weighted boundedness in $L^{q}\left(\mathbb{R}^{n}, \varphi(\cdot, t)\right)$ of $\mathcal{M}_{A}$ with $\varphi \in \mathbb{A}_{q}(A)$ and $q \in(q(\varphi), \infty)$ uniformly in $t \in(0, \infty)$ (see Lemma 2.21), we see that, for all $t \in(0, \infty)$,

$$
\varphi\left(\widetilde{\Omega}_{k}, t\right) \leq\left(2 b^{2 u}\right)^{q} \int_{\mathbb{R}^{n}}\left[\mathcal{M}_{A}\left(\chi_{\Omega_{k}}\right)(x)\right]^{q} \varphi(x, t) d x \lesssim \varphi\left(\Omega_{k}, t\right)
$$

Thus, using $\left\|a_{k}^{\ell}\right\|_{L_{\varphi}^{q}\left(Q_{k}^{\ell}\right)} \lesssim 2^{k}$, (2.23) with the disjointness of $\left\{Q_{k}^{\ell}\right\}_{k \in \mathbb{Z}, \ell},(2.26)$ and the following estimate (see [30, Lemma 5.4] with a slight modification)

$$
\sum_{k \in \mathbb{Z}} \varphi\left(\Omega_{k}, \frac{2^{k}}{\lambda}\right) \lesssim \int_{\mathbb{R}^{n}} \varphi\left(x, \frac{S(f)(x)}{\lambda}\right) d x
$$

we conclude that

$$
\sum_{k \in \mathbb{Z}} \sum_{\ell} \varphi\left(Q_{k}^{\ell}, \frac{\left\|a_{k}^{\ell}\right\|_{L_{\varphi}^{q}\left(Q_{k}^{\ell}\right)}}{\lambda}\right) \lesssim \sum_{k \in \mathbb{Z}} \varphi\left(\Omega_{k}, \frac{2^{k}}{\lambda}\right) \lesssim \int_{\mathbb{R}^{n}} \varphi\left(x, \frac{S(f)(x)}{\lambda}\right) d x
$$

which implies $\|f\|_{H_{A}^{\varphi, q, s}\left(\mathbb{R}^{n}\right)} \lesssim\|S(f)\|_{L^{\varphi}\left(\mathbb{R}^{n}\right)}$.

Step 3. Show $\operatorname{supp} a_{k}^{\ell} \subset B_{k}^{\ell}:=x_{Q_{k}^{\ell}}+B_{v\left[\ell\left(Q_{k}^{\ell}\right)-1\right]+u+3 \sigma}$.

If $x \in \operatorname{supp} a_{k}^{\ell}$, by the definition of $a_{k}^{\ell}$ in (2.21), $a_{k}^{\ell}(x) \neq 0$ implies that there exists $Q \subset Q_{k}^{\ell}$ and $Q \in \mathcal{R}_{k}$ such that $e_{Q}(x) \neq 0$. Recall that, for all $m \in \mathbb{Z}$ and $x \in \mathbb{R}^{n}$,

$$
\theta_{-m}(x):=b^{-m} \theta\left(A^{-m} x\right)
$$


and $\operatorname{supp} \theta \subset B_{0}$. If $e_{Q}(x) \neq 0$, by (2.20) again, there exists $(y, m) \in \widetilde{Q}$ such that $A^{-m}(x-y) \in B_{0}$. Moreover, by (2.18), (2.2) and Lemma 2.9(iv), we further have

$$
x \in y+B_{m} \subset x_{Q}+B_{v \ell(Q)+u}+B_{v[\ell(Q)-1]+u+\sigma} \subset x_{Q}+B_{v[\ell(Q)-1]+u+2 \sigma} .
$$

Thus,

$$
\operatorname{supp} e_{Q} \subset x_{Q}+B_{v[\ell(Q)-1]+u+2 \sigma} .
$$

From this, $a_{k}^{\ell}=\sum_{Q \subset Q_{k}^{\ell}, Q \in \mathcal{R}_{k}} e_{Q}$, Lemma 2.9(iv) and (2.2), we deduce that

$$
\begin{aligned}
\operatorname{supp} a_{k}^{\ell} & \subset \bigcup_{Q \subset Q_{k}^{\ell}, Q \in \mathcal{R}_{k}}\left(x_{Q}+B_{v[\ell(Q)-1]+u+2 \sigma}\right) \\
& \subset x_{Q_{k}^{\ell}}+B_{v \ell\left(Q_{k}^{\ell}\right)+u}+B_{v\left[\ell\left(Q_{k}^{\ell}\right)-1\right]+u+2 \sigma} \\
& \subset x_{Q_{k}^{\ell}}+B_{v\left[\ell\left(Q_{k}^{\ell}\right)-1\right]+u+3 \sigma} .
\end{aligned}
$$

Step 4. Prove $\left\|\widetilde{a}_{k}^{\ell}\right\|_{L_{\varphi}^{q}\left(B_{k}^{\ell}\right)} \leq\left\|\chi_{B_{k}^{\ell}}\right\|_{L^{\varphi}\left(\mathbb{R}^{n}\right)}^{-1}$.

Let $\lambda_{k}^{\ell}:=\widetilde{C} 2^{k}\left\|\chi_{B_{k}^{\ell}}\right\|_{L^{\varphi}\left(\mathbb{R}^{n}\right)}$ and $\widetilde{a}_{k}^{\ell}:=\left(\lambda_{k}^{\ell}\right)^{-1} a_{k}^{\ell}$. Then we have

$$
f=\sum_{k \in \mathbb{Z}} \sum_{\ell} a_{k}^{\ell}=\sum_{k \in \mathbb{Z}} \sum_{\ell} \lambda_{k}^{\ell} \widetilde{a}_{k}^{\ell}
$$

in $\mathcal{S}^{\prime}\left(\mathbb{R}^{n}\right)$. By $\left\|a_{k}^{\ell}\right\|_{L_{\varphi}^{q}\left(Q_{k}^{\ell}\right)} \leq \widetilde{C} 2^{k}$, we see that $\left\|\widetilde{a}_{k}^{\ell}\right\|_{L_{\varphi}^{q}\left(Q_{k}^{\ell}\right)} \leq\left\|\chi_{B_{k}^{\ell}}\right\|_{L^{\varphi}\left(\mathbb{R}^{n}\right)}^{-}$.

Step 5. Show the vanishing moments of $a_{k}^{\ell}$.

By $(2.25)$ and $\varphi\left(\widetilde{\Omega}_{k}, t\right) \lesssim \varphi\left(\Omega_{k}, t\right)$ (see $\left.(2.26)\right)$, we obtain

$$
\begin{aligned}
& \left\|\left[\sum_{Q \in \mathcal{R}_{k}}\left(c_{Q}\right)^{2} \chi_{Q \cap\left(\tilde{\Omega}_{k} \backslash \Omega_{k+1}\right)}\right]^{1 / 2}\right\|_{L^{q}\left(\mathbb{R}^{n}, \varphi(\cdot, t)\right)}^{q} \\
\leq & \int_{\mathbb{R}^{n}}\left\{\chi_{Q_{k}^{\ell} \cap\left(\tilde{\Omega}_{k} \backslash \Omega_{k+1}\right)}(x) \int_{\bigcup_{Q \subset Q_{k}^{\ell}, Q \in \mathcal{R}_{k}} \widetilde{Q}}\left|f * \psi_{-m}(y)\right|^{2} d y \frac{d \sigma(m)}{b^{m}}\right\}^{q / 2} \varphi(x, t) d x \\
\lesssim & 2^{k q} \varphi\left(\Omega_{k}, t\right)<\infty .
\end{aligned}
$$

Take any $N \in \mathbb{N}$ and let $\mathcal{R}_{k, N}:=\left\{Q \in \mathcal{R}_{k}:|\ell(Q)|>N\right\}$. Similar to the estimate of 
(2.24), via replacing $a_{k}^{\ell}$ by $\sum_{Q \subset Q_{k}^{\ell}, Q \in \mathcal{R}_{k, N}} e_{Q}$, we conclude that

$$
\begin{aligned}
& \left.\left\|\sum_{Q \subset Q_{k}^{\ell}, Q \in \mathcal{R}_{k, N}} e_{R}\right\|_{L^{q}\left(\mathbb{R}^{n}, \varphi(\cdot, t)\right)}^{q}\left(c_{Q}\right)^{2} \chi_{Q \cap\left(\tilde{\Omega}_{k} \backslash \Omega_{k+1}\right)}\right]^{1 / 2} \|_{L^{q}\left(\mathbb{R}^{n}, \varphi(\cdot, t)\right)}^{q} \\
\lesssim & \| \sum_{Q \subset Q_{k}^{\ell}, Q \in \mathcal{R}_{k, N}} \int_{\mathbb{R}^{n}} \chi_{Q_{k}^{\ell} \cap\left(\tilde{\Omega}_{k} \backslash \Omega_{k+1}\right)}(x)\left[\int_{\bigcup_{Q \subset Q_{k}^{\ell}, Q \in \mathcal{R}_{k, N}} \widetilde{Q}}\left|f * \psi_{-m}(y)\right|^{2} d y \frac{d \sigma(m)}{b^{m}}\right]^{q / 2} \varphi(x, t) d x .
\end{aligned}
$$

Then, by (2.27) and Lebesgue's dominated convergence theorem, we have

$$
\left\|\sum_{Q \subset Q_{k}^{\ell}, Q \in \mathcal{R}_{k, N}} e_{Q}\right\|_{L^{q}\left(\mathbb{R}^{n}, \varphi(\cdot, t)\right)} \rightarrow 0
$$

as $N \rightarrow \infty$, which implies that $a_{k}^{\ell}=\sum_{\substack{Q \subset Q_{k}^{\ell} \\ Q \in \mathcal{R}_{k}}} e_{Q}$ holds true in $L^{q}\left(\mathbb{R}^{n}, \varphi(\cdot, t)\right)$. Moreover, recall that $\theta$ has the vanishing moments $s \geq m(\varphi)$ and so is $e_{Q}$. Let $h(x):=x^{\alpha} \chi_{B_{k}^{\ell}}(x)$ for all $x \in \mathbb{R}^{n}$ with $|\alpha| \leq s$ and $q^{\prime} \in[1, \infty)$ such that $1 / q+1 / q^{\prime}=1$. Obviously,

$$
h \in L^{q^{\prime}}\left(\mathbb{R}^{n},[\varphi(\cdot, t)]^{-q^{\prime} / q}\right) .
$$

Then, by the fact that $\operatorname{supp} a_{k}^{\ell} \subset B_{k}^{\ell},\left(L^{q^{\prime}}\left(\mathbb{R}^{n},[\varphi(\cdot, t)]^{-q^{\prime} / q}\right)\right)^{*}=L^{q}\left(\mathbb{R}^{n}, \varphi(\cdot, t)\right)$ and supp $e_{Q} \subset B_{k}^{\ell}$, we obtain

$$
\int_{\mathbb{R}^{n}} a_{k}^{\ell}(x) x^{\alpha} d x=\left\langle a_{k}^{\ell}, h\right\rangle=\sum_{Q \subset Q_{k}^{\ell}, Q \in \mathcal{R}_{k}}\left\langle e_{Q}, h\right\rangle=\sum_{Q \subset Q_{k}^{\ell}, Q \in \mathcal{R}_{k}} \int_{\mathbb{R}^{n}} e_{Q}(x) x^{\alpha} d x=0 .
$$

Thus, $a_{k}^{\ell}$ has the vanishing moments up to order $s$. Combining Steps 3 through 5, we find that $a_{k}^{\ell}$ is a multiple of a $(\varphi, q, s)$-atom associated to $B_{k}^{\ell}$. This finishes the proof of Theorem 2.8.

Corollary 2.22. Let $\varphi$ be an anisotropic growth function as in Definition 2.3. Then $H_{S, A}^{\varphi}\left(\mathbb{R}^{n}\right)$ is well defined. Precisely, if $\psi_{1}, \psi_{2} \in \mathcal{S}\left(\mathbb{R}^{n}\right)$ are as in Definition 2.7, then $H_{S_{\psi_{1}}, A}^{\varphi}\left(\mathbb{R}^{n}\right)=H_{S_{\psi_{2}}, A}^{\varphi}\left(\mathbb{R}^{n}\right)$ with equivalent (quasi-)norms, where $S_{\psi_{1}}$ and $S_{\psi_{2}}$ are defined, respectively, as in (2.7) via replacing $\psi$ by $\psi_{1}$ or $\psi_{2}$.

\section{Littlewood-paley $g$-Function and $g_{\lambda}^{*}$-Function Characterizations of $H_{A}^{\varphi}\left(\mathbb{R}^{n}\right)$}

In this section, we establish the Littlewood-Paley $g$-function and $g_{\lambda}^{*}$-function characterizations of $H_{A}^{\varphi}\left(\mathbb{R}^{n}\right)$ via first establishing an anisotropic Peetre's inequality of Musielak-Orlicz type. The following theorem is the first main result of this section. 
Theorem 3.1. Let $\varphi$ be an anisotropic growth function as in Definition 2.3. Then

$$
H_{A}^{\varphi}\left(\mathbb{R}^{n}\right)=H_{g, A}^{\varphi}\left(\mathbb{R}^{n}\right)
$$

with equivalent (quasi-)norms.

To prove Theorem 3.1, we begin with recalling some notation and establishing several technical lemmas. For any $j \in \mathbb{Z}$ and $k \in \mathbb{Z}^{n}$, let $Q_{j, k}:=A^{-j}\left([0,1)^{n}+k\right)$, $\mathcal{Q}_{j}:=\left\{Q_{j, k}: k \in \mathbb{Z}^{n}\right\}$ and $\widetilde{\mathcal{Q}}:=\bigcup_{j \in \mathbb{Z}} \mathcal{Q}_{j}$. Recall that $Q_{j, k}$ for $j \in \mathbb{Z}$ and $k \in \mathbb{Z}^{n}$ is called a dilated cube (see, for example, [7, p. 1475]). Obviously, for all $k_{1}, k_{2} \in \mathbb{Z}^{n}$ and $k_{1} \neq k_{2},\left|Q_{j, k_{1}} \cap Q_{j, k_{2}}\right|=0$.

In what follows, for any $x \in \mathbb{R}^{n}$ and $r \in(0, \infty)$, let

$$
B_{\rho}(x, r):=\left\{y \in \mathbb{R}^{n}: \rho(x-y)<r\right\} .
$$

For any dilated cube $Q_{j, k}$, denoting by $c_{Q_{j, k}}$ its center, via [6, Lemma 2.9(a)], we know that there exists a positive integer $j_{0}:=j_{(A, n)}$, depending on $A$ and $n$, such that, for any $x \in Q$,

$$
B_{\rho}\left(c_{Q_{j, k}}, b^{-j_{0}-j}\right) \subset Q_{j, k} \subset B_{\rho}\left(x, b^{j_{0}-j}\right) .
$$

For any function $\Phi$ on $\mathbb{R}^{n}$ and $Q:=Q_{j, k}$, the left corner $A^{-j} k$ in $Q_{j, k}$ is denoted by $x_{Q_{j, k}}$, and let $x_{Q}:=x_{Q_{j, k}}$ and, for all $x \in \mathbb{R}^{n}$,

$$
\Phi_{Q}(x):=|Q|^{1 / 2} \Phi_{j}\left(x-x_{Q}\right) .
$$

We need the following discrete Calderón reproducing formula which is an anisotropic version of [18, Theorem 6.16].

Lemma 3.2. Let $\Psi, \Phi \in \mathcal{S}\left(\mathbb{R}^{n}\right)$ such that $\operatorname{supp} \widehat{\Phi}$, supp $\widehat{\Psi} \subset[-1,1]^{n} \backslash\left\{\overrightarrow{0}_{n}\right\}$ and

$$
\sum_{j \in \mathbb{Z}} \overline{\widehat{\Phi}\left(\left(A^{*}\right)^{j} \xi\right)} \widehat{\Psi}\left(\left(A^{*}\right)^{j} \xi\right)=1 \quad \text { for all } \xi \in \mathbb{R}^{n} \backslash\left\{\overrightarrow{0}_{n}\right\}
$$

where $A^{*}$ denotes the transpose of $A$. Then, for all $f \in \mathcal{S}_{0}^{\prime}\left(\mathbb{R}^{n}\right)$,

$$
f(\cdot)=\sum_{Q \in \widetilde{\mathcal{Q}}}\left\langle f, \Phi_{Q}\right\rangle \Psi_{Q}(\cdot)=\sum_{j \in \mathbb{Z}} \sum_{Q \in \mathcal{Q}_{j}} b^{-j} f * \widetilde{\Phi}_{j}\left(x_{Q}\right) \Psi_{j}\left(\cdot-x_{Q}\right)
$$

holds true in $\mathcal{S}^{\prime}\left(\mathbb{R}^{n}\right)$, where $\widetilde{\Phi}(\cdot):=\overline{\Phi(-\cdot)}$, and $\Phi_{Q}$ and $\Psi_{Q}$ are defined as in (3.2).

Proof. For any $f \in \mathcal{S}_{0}^{\prime}\left(\mathbb{R}^{n}\right)$ and $\Phi, \Psi$ as in Lemma 3.2, by [9, Lemma 2.12(i)], we have $f=\sum_{j \in \mathbb{Z}} f * \widetilde{\Phi}_{j} * \Psi_{j}$ in $\mathcal{S}^{\prime}\left(\mathbb{R}^{n}\right)$, which, combined with the fact that

$$
f * \widetilde{\Phi}_{j} * \Psi_{j}(\cdot)=\sum_{Q \in \mathcal{Q}_{j}} b^{-j} f * \widetilde{\Phi}_{j}\left(x_{Q}\right) \Psi_{j}\left(\cdot-x_{Q}\right)
$$


in $\mathcal{S}^{\prime}\left(\mathbb{R}^{n}\right)$ (see the proof of [6, Lemma 2.8, pp. 1478-1479]), then completes the proof of Lemma 3.2.

Here, we point out that the condition supp $\widehat{\Psi} \subset[-1,1]^{n} \backslash\left\{\overrightarrow{0}_{n}\right\}$ in Lemma 3.2 implies that $\int_{\mathbb{R}^{n}} \Psi(x) x^{\alpha} d x=0$ for all multi-indices $\alpha$.

For any $j, j^{\prime} \in \mathbb{Z}$ and $\psi, \phi \in \mathcal{S}\left(\mathbb{R}^{n}\right)$, by [9, Lemma 5.4(i)] with

$$
\psi_{j^{\prime}} * \phi_{j}(\cdot)=b^{j}\left(\psi_{j^{\prime}-j} * \phi\right)\left(A^{j} \cdot\right)
$$

we have the following Lemma 3.3, the details being omitted.

Lemma 3.3. Let $s, M \in \mathbb{N}$ and $\psi, \phi \in \mathcal{S}\left(\mathbb{R}^{n}\right)$ which have the vanishing moments up to order $s$. Then there exists a positive constant $C_{(s, M)}$, depending on $s$ and $M$, such that, for all $j, j^{\prime} \in \mathbb{Z}$ with $j^{\prime} \geq j$ and $x \in \mathbb{R}^{n}$,

$$
\left|\psi_{j^{\prime}} * \phi_{j}(x)\right| \leq C_{(s, M)} b^{j-\left(j^{\prime}-j\right)(s+1) \zeta_{-}}\left[1+\rho\left(A^{j} x\right)\right]^{-M},
$$

where $\zeta_{-}:=\ln \left(\lambda_{-}\right) / \ln b$.

Definition 3.4. Let $r, \lambda \in(0, \infty)$. For any sequence $\mathbf{s}:=\left\{s_{Q}\right\}_{Q \in \widetilde{\mathcal{Q}}} \subset \mathbb{C}$, its majorant sequence $s_{r, \lambda}^{*}:=\left\{\left(s_{r, \lambda}^{*}\right)_{Q}\right\}_{Q \in \widetilde{\mathcal{Q}}}$, is defined, by setting, for all $Q \in \widetilde{\mathcal{Q}}$,

$$
\left(s_{r, \lambda}^{*}\right)_{Q}:=\left\{\sum_{P \in \widetilde{\mathcal{Q}},|P|=|Q|} \frac{\left|s_{P}\right|^{r}}{\left[1+|Q|^{-1} \rho\left(x_{Q}-x_{P}\right)\right]^{\lambda}}\right\}^{\frac{1}{r}} .
$$

The following Lemma 3.5 is just [5, Lemma 6.2].

Lemma 3.5. Let $j \in \mathbb{Z}, a, r \in(0, \infty)$ with $a \leq r$ and $\lambda \in(r / a, \infty)$. Then there exists a positive constant $C$ such that, for any sequence $\mathbf{s}:=\left\{s_{Q}\right\}_{Q \in \widetilde{\mathcal{Q}}} \subset \mathbb{C}$,

$$
\sum_{|Q|=b^{-j}}\left(s_{r, \lambda}^{*}\right)_{Q} \chi_{Q} \leq C\left[\mathcal{M}_{A}\left(\sum_{|Q|=b^{-j}}\left|s_{Q}\right|^{a} \chi_{Q}\right)\right]^{\frac{1}{a}} .
$$

The following Lemma 3.6 is an anisotropic version of the Musielak-Orlicz FeffermanStein vector-valued inequality from [34, Theorem 2.10], whose proof is also an obvious modification of the proof of [34, Theorem 2.10], the details being omitted.

Lemma 3.6. Let $r \in(1, \infty], \varphi$ be a Musielak-Orlicz function with the uniformly lower type $p_{\varphi}^{-}$and the uniformly upper type $p_{\varphi}^{+}, q \in(1, \infty)$ and $\varphi \in \mathbb{A}_{q}(A)$. If $q(\varphi)<p_{\varphi}^{-} \leq p_{\varphi}^{+}<\infty$, then there exists a positive constant $C$ such that, for all $\left\{f_{j}\right\}_{j \in \mathbb{Z}} \in L^{\varphi}\left(\ell^{r}, \mathbb{R}^{n}\right)$,

$$
\int_{\mathbb{R}^{n}} \varphi\left(x,\left\{\sum_{j \in \mathbb{Z}}\left[\mathcal{M}_{A}\left(f_{j}\right)(x)\right]^{r}\right\}^{\frac{1}{r}}\right) d x \leq C \int_{\mathbb{R}^{n}} \varphi\left(x,\left\{\sum_{j \in \mathbb{Z}}\left|f_{j}(x)\right|^{r}\right\}^{\frac{1}{r}}\right) d x .
$$


Lemma 3.7. Let $\varphi$ be an anisotropic growth function as in Definition 2.3. Then, for any $r \in(0, \infty)$ and $\lambda \in(\max \{1, r / 2, r q(\varphi) / i(\varphi)\}, \infty)$, there exists a positive constant $C$ such that, for all $\mathbf{s}:=\left\{s_{Q}\right\}_{Q \in \widetilde{\mathcal{Q}}}$,

$$
\int_{\mathbb{R}^{n}} \varphi\left(x,\left[\sum_{Q \in \widetilde{\mathcal{Q}}}\left\{\left(s_{r, \lambda}^{*}\right)_{Q}\right\}^{2} \chi_{Q}(x)\right]^{\frac{1}{2}}\right) d x \leq C \int_{\mathbb{R}^{n}} \varphi\left(x,\left[\sum_{Q \in \widetilde{\mathcal{Q}}}\left|s_{Q}\right|^{2} \chi_{Q}(x)\right]^{\frac{1}{2}}\right) d x .
$$

Proof. We show this lemma by borrowing some ideas from the proof of [5, Lemma 3.3]. Let $r \in(0, \infty)$ and $\lambda \in(\max \{1, r / 2, r q(\varphi) / i(\varphi)\}, \infty)$. If $r<$ $\min \{2, i(\varphi) / q(\varphi)\}$, we let $a:=r$. Otherwise, if $r \geq \min \{2, i(\varphi) / q(\varphi)\}$, we choose $a$ such that $r / \lambda<a<\min \{r, 2, i(\varphi) / q(\varphi)\}$. It is possible to choose such an $a$, since $\lambda>\max \{1, r / 2, r q(\varphi) / i(\varphi)\}$ implies $r / \lambda<\min \{r, 2, i(\varphi) / q(\varphi)\}$. In both cases, we find that

$$
0<a \leq r<\infty, \quad \lambda>\frac{r}{a}, \quad \frac{2}{a}>1 \quad \text { and } \quad \frac{i(\varphi)}{a}>q(\varphi) .
$$

For the above last inequality, by choosing $p \in(0, i(\varphi))$ close to $i(\varphi)$, we further obtain $p / a>q(\varphi)$. Therefore, by Lemma 3.5, we conclude that

$$
\begin{aligned}
& \int_{\mathbb{R}^{n}} \varphi\left(x,\left\{\sum_{j \in \mathbb{Z}}\left[\sum_{|Q|=b^{-j}}\left(s_{r, \lambda}^{*}\right)_{Q} \chi_{Q}(x)\right]^{2}\right\}^{\frac{1}{2}}\right) d x \\
\lesssim & \int_{\mathbb{R}^{n}} \varphi\left(x,\left\{\left(\sum_{j \in \mathbb{Z}}\left[\mathcal{M}_{A}\left(\sum_{|Q|=b^{-j}}\left|s_{Q}\right|^{a} \chi_{Q}\right)(x)\right]^{\frac{2}{a}}\right)^{\frac{a}{2}}\right\}^{\frac{1}{a}}\right) d x .
\end{aligned}
$$

Moreover, let $\widetilde{\varphi}(x, t):=\varphi\left(x, t^{1 / a}\right)$ for all $x \in \mathbb{R}^{n}$ and $t \in(0, \infty)$. From the fact that $\varphi$ is of uniformly upper type 1 and uniformly lower type $p$, it follows that $\widetilde{\varphi}$ is of uniformly upper type $1 / a$ and uniformly lower type $p / a$. Then, applying Lemma 3.6 with $1 / a>p / a>q(\varphi)$ to (3.3), we obtain the desired inequality in Lemma 3.7 and hence complete the proof of Lemma 3.7.

The following Lemma 3.8 comes from [6, p. 423].

Lemma 3.8. For any $f \in \mathcal{S}^{\prime}\left(\mathbb{R}^{n}\right), \Phi \in \mathcal{S}\left(\mathbb{R}^{n}\right)$ and supp $\widehat{\Phi}$ being compact and bounded away from the origin, the sequences $\sup (f):=\left\{\sup _{Q}(f)\right\}_{Q \in \widetilde{\mathcal{Q}}}$ and $\inf (f):=$ $\left\{\inf _{Q}(f)\right\}_{Q \in \widetilde{\mathcal{Q}}}$ are defined, respectively, by setting, for any $Q \in \widetilde{\mathcal{Q}}$ with $|Q|=b^{-j}$ for some $j \in \mathbb{Z}$,

$$
\sup _{Q}(f):=\sup _{y \in Q}\left|f * \widetilde{\Phi}_{j}(y)\right|
$$


and

$$
\inf _{Q}(f):=\inf \left\{\sup _{y \in P}\left|f * \widetilde{\Phi}_{j}(y)\right|:|P \cap Q| \neq 0,|Q| /|P|=b^{\gamma}\right\},
$$

where $\gamma \in \mathbb{N}$. Then, for any $\lambda, r \in(0, \infty)$ and sufficient large $\gamma \in \mathbb{N}$, there exists a positive constant $C$ such that, for all $Q \in \widetilde{\mathcal{Q}}$,

$$
\left.\sup _{Q}(f)\right)_{r, \lambda}^{*} \leq C\left(\inf _{Q}(f)\right)_{r, \lambda}^{*} .
$$

Proof of Theorem 3.1. Let $(\varphi, q, s)$ be admissible as in Definition 2.11. By repeating the proof of Lemma 2.18 with a slight modification, we easily obtain $H_{A}^{\varphi, q, s}\left(\mathbb{R}^{n}\right) \subset$ $H_{g, A}^{\varphi}\left(\mathbb{R}^{n}\right)$ with continuous inclusion. From this, $H_{A}^{\varphi, q, s}\left(\mathbb{R}^{n}\right) \subset \mathcal{S}_{0}^{\prime}\left(\mathbb{R}^{n}\right)$ and

$$
H_{A}^{\varphi, q, s}\left(\mathbb{R}^{n}\right)=H_{A}^{\varphi}\left(\mathbb{R}^{n}\right)
$$

with equivalent (quasi-)norms (see Lemma 2.13), it follows that $H_{A}^{\varphi}\left(\mathbb{R}^{n}\right) \subset H_{g, A}^{\varphi}\left(\mathbb{R}^{n}\right)$ with continuous inclusion. Conversely, to prove $H_{g, A}^{\varphi}\left(\mathbb{R}^{n}\right) \subset H_{A}^{\varphi}\left(\mathbb{R}^{n}\right)$, since

$$
\|\cdot\|_{H_{A}^{\varphi}\left(\mathbb{R}^{n}\right)} \sim\|\cdot\|_{H_{S, A}^{\varphi}\left(\mathbb{R}^{n}\right)},
$$

we only need to show that, for all $f \in H_{g, A}^{\varphi}\left(\mathbb{R}^{n}\right),\|S(f)\|_{L^{\varphi}\left(\mathbb{R}^{n}\right)} \lesssim\|g(f)\|_{L^{\varphi}\left(\mathbb{R}^{n}\right)}$.

Let $\Phi, \Psi \in \mathcal{S}\left(\mathbb{R}^{n}\right)$ be as in Lemma 3.2 and let $\psi \in \mathcal{S}\left(\mathbb{R}^{n}\right)$ have the vanishing moments up to order $s$ to be fixed later. For any $M \in \mathbb{N}$ to be fixed later, $j \in \mathbb{Z}, f \in$ $\mathcal{S}_{0}^{\prime}\left(\mathbb{R}^{n}\right), x \in \mathbb{R}^{n}$ and $y \in x+B_{-j}$, by Lemmas 3.2 and 3.3, we have

$$
\begin{aligned}
\left|f * \psi_{j}(y)\right| \lesssim & \sum_{j^{\prime} \in \mathbb{Z}} \sum_{Q \in \mathcal{Q}_{j^{\prime}}} b^{-j^{\prime}}\left|f * \widetilde{\Phi}_{j^{\prime}}\left(x_{Q}\right) \Psi_{j^{\prime}} * \psi_{j}\left(y-x_{Q}\right)\right| \\
\lesssim & \sum_{j^{\prime} \leq j} \sum_{Q \in \mathcal{Q}_{j^{\prime}}} \frac{b^{-\left(j-j^{\prime}\right)(s+1) \zeta_{-}}}{\left[1+\rho\left(A^{j^{\prime}}\left(y-x_{Q}\right)\right)\right]^{M}}\left|\left(f * \widetilde{\Phi}_{j^{\prime}}\right)\left(x_{Q}\right)\right| \\
& +\sum_{j^{\prime}>j} \sum_{Q \in \mathcal{Q}_{j^{\prime}}} \frac{b^{-\left(j^{\prime}-j\right)\left[1+(s+1) \zeta_{-}\right]}}{\left[1+\rho\left(A^{j}\left(y-x_{Q}\right)\right)\right]^{M}}\left|\left(f * \widetilde{\Phi}_{j^{\prime}}\right)\left(x_{Q}\right)\right|=: \mathrm{I}+\mathrm{II} .
\end{aligned}
$$

For any $m \in \mathbb{Z}_{+}$, when $m=0$, let $A_{m}:=\left\{Q \in \mathcal{Q}_{j^{\prime}}: \rho\left(A^{j^{\prime}}\left(y-x_{Q}\right)\right) \leq b^{m}\right\}$ and, when $m \geq 1$,

$$
A_{m}:=\left\{Q \in \mathcal{Q}_{j^{\prime}}: b^{m-1}<\rho\left(A^{j^{\prime}}\left(y-x_{Q}\right)\right) \leq b^{m}\right\} .
$$

Then, we conclude that

$$
\begin{aligned}
& \sum_{Q \in A_{m}} \frac{b^{-\left(j-j^{\prime}\right)(s+1) \zeta_{-}}}{\left[1+\rho\left(A^{j^{\prime}}\left(y-x_{Q}\right)\right)\right]^{M}}\left|\left(f * \widetilde{\Phi}_{j^{\prime}}\right)\left(x_{Q}\right)\right| \\
\leq & b^{-\left(j-j^{\prime}\right)(s+1) \zeta_{-}-m M} \sum_{Q \in A_{m}}\left|\left(f * \widetilde{\Phi}_{j^{\prime}}\right)\left(x_{Q}\right)\right| .
\end{aligned}
$$


Notice that, for all $z \in Q \in A_{m}$, by Definition 2.1(iii) and (3.1), we see that

$$
\rho(z-y) \leq H\left[\rho\left(z-x_{Q}\right)+\rho\left(x_{Q}-y\right)\right] \leq H\left[b^{j_{0}-j^{\prime}}+b^{m-j^{\prime}}\right]<2 H b^{j_{0}+m-j^{\prime}},
$$

where $j_{0} \in \mathbb{Z}_{+}$is as in (3.1), which implies that

$$
\bigcup_{Q \in A_{m}} Q \subset B_{\rho}\left(y, 2 H b^{j_{0}+m-j^{\prime}}\right):=\left\{z \in \mathbb{R}^{n}: \rho(z-y)<2 H b^{j_{0}+m-j^{\prime}}\right\}
$$

Moreover, if $j^{\prime} \leq j$, using $m, j_{0} \in \mathbb{N}$ and $x \in y+B_{-j}$ which implies $\rho(x-y)<b^{-j}$, we obtain $x \in B_{\rho}\left(y, 2 H b^{j_{0}+m-j^{\prime}}\right)$. Thus, for all $j^{\prime} \leq j$ and $r \in(0,1]$ to be fixed later, by (3.5), we find that

$$
\begin{aligned}
& \sum_{Q \in A_{m}}\left|f * \widetilde{\Phi}_{j^{\prime}}\left(x_{Q}\right)\right| \\
\lesssim & b^{\frac{j^{\prime}}{r}}\left\{\int_{B_{\rho}\left(y, 2 H b^{j_{0}+m-j^{\prime}}\right)} \sum_{Q \in A_{m}}\left|f * \widetilde{\Phi}_{j^{\prime}}\left(x_{Q}\right)\right|^{r} \chi_{Q}(z) d z\right\}^{1 / r} \\
\lesssim & b^{\frac{m}{r}}\left[\mathcal{M}_{A}\left(\left[\sum_{Q \in \mathcal{Q}_{j^{\prime}}}\left|f * \widetilde{\Phi}_{j^{\prime}}\left(x_{Q}\right)\right| \chi_{Q}\right]^{r}\right)(x)\right]^{1 / r},
\end{aligned}
$$

where $\mathcal{M}_{A}$ is as in (2.17).

We choose $M \in \mathbb{N}$ such that $M>1 / r$. Then, by (3.4) and (3.6), we conclude that

$$
\begin{aligned}
\mathrm{I} & \lesssim \sum_{j^{\prime} \leq j} \sum_{m=0}^{\infty} b^{-\left(j-j^{\prime}\right)(s+1) \zeta_{-}-m\left(M-\frac{1}{r}\right)}\left\{\mathcal{M}_{A}\left(\sum_{Q \in \mathcal{Q}_{j^{\prime}}}\left|f * \widetilde{\Phi}_{j^{\prime}}\left(x_{Q}\right)\right|^{r} \chi_{Q}\right)(x)\right\}^{1 / r} \\
& \sim \sum_{j^{\prime} \leq j} b^{-\left(j-j^{\prime}\right)(s+1) \zeta_{-}}\left\{\mathcal{M}_{A}\left(\left[\sum_{Q \in \mathcal{Q}_{j^{\prime}}}\left|f * \widetilde{\Phi}_{j^{\prime}}\left(x_{Q}\right)\right| \chi_{Q}\right]^{r}\right)(x)\right\}^{1 / r} .
\end{aligned}
$$

Similar to the proof of I, by choosing $M, s \in \mathbb{N}$ such that $M>1 / r$ and

$$
1+(s+1) \zeta_{-}-1 / r>0
$$

we also have

$$
\begin{aligned}
\mathrm{II} & \lesssim \sum_{j^{\prime}>j} \sum_{m=0}^{\infty} b^{-\left(j^{\prime}-j\right)\left[1+(s+1) \zeta_{-}-\frac{1}{r}\right]-m\left(M-\frac{1}{r}\right)}\left\{\mathcal{M}_{A}\left(\left[\sum_{Q \in \mathcal{Q}_{j^{\prime}}}\left|f * \widetilde{\Phi}_{j^{\prime}}\left(x_{Q}\right)\right| \chi_{Q}\right]^{r}\right)(x)\right\}^{1 / r} \\
& \sim \sum_{j^{\prime}>j} b^{-\left(j^{\prime}-j\right)\left[1+(s+1) \zeta_{-}-\frac{1}{r}\right]}\left\{\mathcal{M}_{A}\left(\left[\sum_{Q \in \mathcal{Q}_{j^{\prime}}}\left|f * \widetilde{\Phi}_{j^{\prime}}\left(x_{Q}\right)\right| \chi_{Q}\right]^{r}\right)(x)\right\}^{1 / r} .
\end{aligned}
$$


Combining the estimates of I and II, for any given $j \in \mathbb{Z}, x \in \mathbb{R}^{n}$ and $y \in x+B_{-j}$, we further conclude that

$\left|f * \psi_{j}(y)\right| \lesssim \sum_{j^{\prime} \in \mathbb{Z}} b^{-\left|j-j^{\prime}\right|\left[1-\frac{1}{r}+(s+1) \zeta_{-}\right]}\left\{\mathcal{M}_{A}\left(\left[\sum_{Q \in \mathcal{Q}_{j^{\prime}}}\left|f * \widetilde{\Phi}_{j^{\prime}}\left(x_{Q}\right)\right| \chi_{Q}\right]^{r}\right)(x)\right\}^{1 / r}$

Consequently, it holds true that, for all $x \in \mathbb{R}^{n}$,

$$
\begin{aligned}
& {[S(f)(x)]^{2} } \\
= & \sum_{j \in \mathbb{Z}} b^{j} \int_{x+B_{-j}}\left|f * \psi_{j}(y)\right|^{2} d y \\
\lesssim & \sum_{j \in \mathbb{Z}}\left\{\sum_{j^{\prime} \in \mathbb{Z}} b^{-\left|j^{\prime}-j\right|\left[1-\frac{1}{r}+(s+1) \zeta_{-}\right]}\left[\mathcal{M}_{A}\left(\left[\sum_{Q \in \mathcal{Q}_{j^{\prime}}}\left|f * \widetilde{\Phi}_{j^{\prime}}\left(x_{Q}\right)\right| \chi_{Q}\right]^{r}\right)(x)\right]^{\frac{1}{r}}\right\}^{2} .
\end{aligned}
$$

Since $1-1 / r+(s+1) \zeta_{-}>0$, by the Hölder inequality, we conclude that

$$
\begin{aligned}
& \sum_{j^{\prime} \in \mathbb{Z}} b^{-\left|j^{\prime}-j\right|\left[1-\frac{1}{r}+(s+1) \zeta_{-}\right]}\left[\mathcal{M}_{A}\left(\left[\sum_{Q \in \mathcal{Q}_{j^{\prime}}}\left|f * \widetilde{\Phi}_{j^{\prime}}\left(x_{Q}\right)\right| \chi_{Q}\right]^{r}\right)(x)\right]^{\frac{1}{r}} \\
\lesssim & \left\{\sum_{j^{\prime} \in \mathbb{Z}} b^{-\left|j^{\prime}-j\right|\left[1-\frac{1}{r}+(s+1) \zeta_{-}\right]}\left[\mathcal{M}_{A}\left(\left[\sum_{Q \in \mathcal{Q}_{j^{\prime}}}\left|f * \widetilde{\Phi}_{j^{\prime}}\left(x_{Q}\right)\right| \chi_{Q}\right]^{r}\right)(x)\right]^{\frac{2}{r}}\right\}^{\frac{1}{2}},
\end{aligned}
$$

which further implies that

$$
S(f)(x) \lesssim\left\{\sum_{j^{\prime} \in \mathbb{Z}}\left[\mathcal{M}_{A}\left(\left[\sum_{Q \in \mathcal{Q}_{j^{\prime}}}\left|f * \widetilde{\Phi}_{j^{\prime}}\left(x_{Q}\right)\right| \chi_{Q}\right]^{r}\right)(x)\right]^{\frac{2}{r}}\right\}^{\frac{1}{2}}
$$

Choose $M \in \mathbb{N}$ large enough such that $r \in(1 / M, p / q(\varphi))$. Let $\widetilde{\varphi}(x, t):=$ $\varphi\left(x, t^{1 / r}\right)$ for all $x \in \mathbb{R}^{n}$ and $t \in(0, \infty)$. From the fact that $\varphi$ is of uniformly upper type 1 and uniformly lower type $p$, it follows that $\widetilde{\varphi}$ is of uniformly upper $1 / r$ and uniformly lower type $p / r$. Then, by Lemma 3.6 with $1 / r>p / r>q(\varphi)$, we have

$$
\int_{\mathbb{R}^{n}} \varphi\left(x,\left\{\sum_{j \in \mathbb{Z}}\left[\mathcal{M}_{A}\left(\left[f_{j}\right]^{r}\right)(x)\right]^{\frac{2}{r}}\right\}^{\frac{1}{2}}\right) d x \lesssim \int_{\mathbb{R}^{n}} \varphi\left(x,\left[\sum_{j \in \mathbb{Z}}\left|f_{j}(x)\right|^{2}\right]^{\frac{1}{2}}\right) d x
$$


which, together with (3.7), implies that

$$
\begin{aligned}
& \int_{\mathbb{R}^{n}} \varphi(x, S(f)(x)) d x \\
\lesssim & \int_{\mathbb{R}^{n}} \varphi\left(x,\left\{\sum_{j^{\prime} \in \mathbb{Z}}\left[\mathcal{M}_{A}\left(\left[\sum_{Q \in \mathcal{Q}_{j^{\prime}}}\left|f * \widetilde{\Phi}_{j^{\prime}}\left(x_{Q}\right)\right| \chi_{Q}\right]^{r}\right)(x)\right]^{\frac{2}{r}}\right\}^{\frac{1}{2}}\right) d x \\
\lesssim & \int_{\mathbb{R}^{n}} \varphi\left(x,\left\{\sum_{j^{\prime} \in \mathbb{Z}} \sum_{Q \in \mathcal{Q}_{j^{\prime}}}\left[\left|f * \widetilde{\Phi}_{j^{\prime}}\left(x_{Q}\right)\right| \chi_{Q}(x)\right]^{2}\right\}^{\frac{1}{2}}\right) d x .
\end{aligned}
$$

Notice that $s_{Q} \leq\left(s_{r, \lambda}^{*}\right)_{Q}$ for any $Q \in \widetilde{\mathcal{Q}}$ and $r, \lambda \in(0, \infty)$. Then, from this, (3.8), Lemmas 3.8 and 3.7 with $r \in(0, \infty), \lambda \in(\max \{1, r / 2, r q(\varphi) / i(\varphi)\}, \infty)$, it follows that, for some $\gamma \in \mathbb{N}$ large enough,

$$
\begin{aligned}
\int_{\mathbb{R}^{n}} \varphi(x, S(f)(x)) d x & \lesssim \int_{\mathbb{R}^{n}} \varphi\left(x,\left[\sum_{Q \in \widetilde{\mathcal{Q}}}\left\{\left(\sup _{Q}(f)_{r, \lambda}^{*}\right)\right\}^{2} \chi_{Q}(x)\right]^{\frac{1}{2}}\right) d x \\
& \lesssim \int_{\mathbb{R}^{n}} \varphi\left(x,\left[\sum_{Q \in \widetilde{\mathcal{Q}}}\left\{\left(\inf _{Q}(f)_{r, \lambda}^{*}\right)\right\}^{2} \chi_{Q}(x)\right]^{\frac{1}{2}}\right) d x \\
& \lesssim \int_{\mathbb{R}^{n}} \varphi\left(x,\left[\sum_{Q \in \widetilde{\mathcal{Q}}}\left\{\inf _{Q}(f)\right\}^{2} \chi_{Q}(x)\right]^{\frac{1}{2}}\right) d x
\end{aligned}
$$

Moreover, for any $P \in \widetilde{\mathcal{Q}}$ with $|P|=b^{-i}$ and $s_{P}:=\inf _{y \in P}\left|f * \widetilde{\Phi}_{i-\gamma}(y)\right|$, by the proof of [6, Lemma 8.4, p. 422], we find that, $\inf _{Q}(f)=\sup \left\{s_{P}:|Q| /|P|=b^{\gamma}, P \in \widetilde{\mathcal{Q}}\right\}$ and, for all $x \in \mathbb{R}^{n}$,

$$
\sum_{|Q|=b^{-j}} \inf _{Q}(f) \chi_{Q}(x) \lesssim b^{\frac{\gamma \lambda}{r}} \sum_{|P|=b^{-j-\gamma}}\left(s_{r, \lambda}^{*}\right)_{P} \chi_{P}(x) .
$$

Combining this, (3.9) and Lemma 3.7, we find that

$$
\begin{aligned}
\int_{\mathbb{R}^{n}} \varphi(x, S(f)(x)) d x & \lesssim \int_{\mathbb{R}^{n}} \varphi\left(x,\left[\sum_{j \in \mathbb{Z}} \sum_{|P|=b^{-j-\gamma}}\left|\left(s_{r, \lambda}^{*}\right)_{P}\right|^{2} \chi_{P}(x)\right]^{\frac{1}{2}}\right) d x \\
& \lesssim \int_{\mathbb{R}^{n}} \varphi\left(x,\left[\sum_{i \in \mathbb{Z}} \sum_{|P|=b^{-i}}\left|s_{P}\right|^{2} \chi_{P}(x)\right]^{\frac{1}{2}}\right) d x
\end{aligned}
$$




$$
\lesssim \int_{\mathbb{R}^{n}} \varphi(x, g(f)(x)) d x
$$

which implies that $\|S(f)\|_{L^{\varphi}\left(\mathbb{R}^{n}\right)} \lesssim\|g(f)\|_{L^{\varphi}\left(\mathbb{R}^{n}\right)}$ and hence completes the proof of Theorem 3.1.

Next, motivated by Folland and Stein [17], Aguilera and Segovia [1] and Liang et al. [34], we consider the Littlewood-Paley $g_{\lambda}^{*}$-function characterization of $H_{A}^{\varphi}\left(\mathbb{R}^{n}\right)$ as follows.

Theorem 3.9. Let $q \in[1, \infty), \varphi \in \mathbb{A}_{q}(A)$ be an anisotropic growth function and $\lambda \in(2 q / p, \infty)$. Then there exists a positive constant $C:=C_{(\varphi, q)}$, depending on $\varphi$ and $q$, such that, for all $f \in \mathcal{S}^{\prime}\left(\mathbb{R}^{n}\right)$,

$$
\|S(f)\|_{L^{\varphi}\left(\mathbb{R}^{n}\right)} \leq\left\|g_{\lambda}^{*}(f)\right\|_{L^{\varphi}\left(\mathbb{R}^{n}\right)} \leq C\|S(f)\|_{L^{\varphi}\left(\mathbb{R}^{n}\right)}
$$

and, furthermore, $H_{S, A}^{\varphi}\left(\mathbb{R}^{n}\right)=H_{g_{\lambda}^{*}, A}^{\varphi}\left(\mathbb{R}^{n}\right)=H_{A}^{\varphi}\left(\mathbb{R}^{n}\right)$ with equivalent (quasi-)norms.

To show Theorem 3.9, we need the following two technical lemmas, whose proofs are motivated by Aguilera and Segovia [1, Lemmas 1 and 2]. Let us begin with introducing some notation. For any subset $E \subset \mathbb{R}^{n}$ and $k_{0} \in \mathbb{N}$, let $\Gamma_{k_{0}}(E):=\bigcup_{x \in E} \Gamma_{k_{0}}(x)$, where

$$
\Gamma_{k_{0}}(x):=\left\{(y, k) \in \mathbb{R}^{n} \times \mathbb{Z}: y \in x+B_{k_{0}+k}\right\} .
$$

The proof of the following lemma is a slight modification of the proof of [30, Lemma 4.1(i)], the details being omitted.

Lemma 3.10. Let $\varphi$ be an anisotropic growth function as in Definition 2.3. Then there exists a positive constant $C$ such that, for all $\left(x, t_{j}\right) \in \mathbb{R}^{n} \times[0, \infty)$ with $j \in \mathbb{N}$,

$$
\varphi\left(x, \sum_{j=1}^{\infty} t_{j}\right) \leq C \sum_{j=1}^{\infty} \varphi\left(x, t_{j}\right) .
$$

Lemma 3.11. Suppose $E$ is an open subset of $\mathbb{R}^{n}$. Let

$$
U:=U_{k_{0}}:=\left\{x \in \mathbb{R}^{n}: \mathcal{M}_{A}\left(\chi_{E}\right)(x)>b^{-2 \sigma-k_{0}}\right\},
$$

where $\sigma \in \mathbb{Z}_{+}$is as in (2.2). Then

(i) $\Gamma_{k_{0}}\left(U^{\complement}\right) \subset \Gamma_{0}\left(E^{\complement}\right)$ for all $k_{0} \in \mathbb{N}$;

(ii) If $(z, k) \in \Gamma_{k_{0}}\left(U^{\complement}\right)$, then $\left|z+B_{k}\right| \leq\left(1-b^{-\sigma}\right)^{-1}\left|\left(z+B_{k}\right) \cap E^{\complement}\right|$.

Proof. If $\Gamma_{k_{0}}\left(U^{\complement}\right)=\emptyset$, then (i) is obviously true, or else $\Gamma_{k_{0}}\left(U^{\complement}\right) \neq \emptyset$, then $U^{\complement} \varsubsetneqq \mathbb{R}^{n}$. For any $(z, k) \in \Gamma_{k_{0}}\left(U^{\complement}\right)$, if $z \in E^{\complement}$, then we have $(z, k) \in \Gamma_{0}\left(E^{\complement}\right)$. If $z \in E$, let us call $b^{m_{0}}$ the distance from $z$ to the closed and non-empty set $E^{\complement}$ associated 
to $\rho$, namely, there exists some $m_{0} \in \mathbb{Z}$ such that $b^{m_{0}}=\inf \left\{\rho\left(z-z^{\prime}\right): z^{\prime} \in E^{\complement}\right\}$, which implies that

$$
z+B_{m_{0}} \subset E .
$$

Moreover, since $(z, k) \in \Gamma_{k_{0}}\left(U^{\complement}\right)$, it follows that there exists some $y \in U^{\complement}$ such that $(z, k) \in \Gamma_{k_{0}}(y)$, namely, $z \in y+B_{k_{0}+k}$. Hence, by (2.2), we obtain

$$
z+B_{m_{0}} \subset y+B_{k_{0}+k}+B_{m_{0}} \subset y+B_{\max \left\{k_{0}+k, m_{0}\right\}+\sigma} .
$$

From this, (3.11) and $y \in U^{\complement}$, it follows that

$$
b^{m_{0}}=\left|z+B_{m_{0}}\right| \leq\left|\left(y+B_{\max \left\{k_{0}+k, m_{0}\right\}+\sigma}\right) \cap E\right| \leq b^{\max \left\{k_{0}+k, m_{0}\right\}-\sigma-k_{0}},
$$

which implies $m_{0}<k$, since $k_{0}, \sigma \in \mathbb{N}$. Then, by the definition of $b^{m_{0}}$, there exists some $x \in E^{\complement}$ satisfying $x \in z+B_{k}$, which means $(z, k) \in \Gamma_{0}\left(E^{\complement}\right)$. This proves (i).

Next, we prove (ii). If $(z, k) \in \Gamma_{k_{0}}\left(U^{\complement}\right)$, then there exists some $y \in U^{\complement}$ such that $z \in y+B_{k_{0}+k}$. Then, by (2.2) and $k_{0} \in \mathbb{N}$, we obtain $z+B_{k} \subset y+B_{k_{0}+k}+B_{k} \subset$ $y+B_{k_{0}+k+\sigma}$ and, since $y \in U^{\complement}$, it follows that

$$
\left|\left(z+B_{k}\right) \cap E\right| \leq\left|\left(y+B_{k_{0}+k+\sigma}\right) \cap E\right| \leq b^{-\sigma}\left|z+B_{k}\right| .
$$

Using this and $\left|z+B_{k}\right|=\left|\left(z+B_{k}\right) \cap E\right|+\left|\left(z+B_{k}\right) \cap E^{\complement}\right|$, we find that

$$
\left|z+B_{k}\right| \leq\left(1-b^{-\sigma}\right)^{-1}\left|\left(z+B_{k}\right) \cap E^{\complement}\right|,
$$

which completes the proof of (ii) and hence Lemma 3.11.

Now we introduce the following variant of the anisotropic Lusin-area function $S$. For all $k_{0} \in \mathbb{N}, \psi \in \mathcal{S}\left(\mathbb{R}^{n}\right), f \in \mathcal{S}^{\prime}\left(\mathbb{R}^{n}\right)$ and $x \in \mathbb{R}^{n}$, let

$$
S_{k_{0}}(f)(x):=\left\{\sum_{k \in \mathbb{Z}} b^{-\left(k_{0}+k\right)} \int_{x+B_{k_{0}+k}}\left|f * \psi_{-k}(y)\right|^{2} d y\right\}^{\frac{1}{2}} .
$$

Lemma 3.12. Let $q \in[1, \infty), \varphi \in \mathbb{A}_{q}(A), k_{0} \in \mathbb{N}$ and $E$ be an open set in $\mathbb{R}^{n}$. If $U:=U_{k_{0}}$ is the set associated to $E$ as in Lemma 3.11, then, for any $k_{0} \in \mathbb{N}$ and $\lambda \in(0, \infty)$, there exists a positive constant $C$ such that, for all $f \in \mathcal{S}^{\prime}\left(\mathbb{R}^{n}\right)$,

$$
b^{k_{0}(1-q)} \int_{U^{\mathrm{C}}}\left[S_{k_{0}}(f)(x)\right]^{2} \varphi(x, \lambda) d x \leq C \int_{E^{\mathrm{C}}}[S(f)(x)]^{2} \varphi(x, \lambda) d x .
$$

Proof. Let $k_{0} \in \mathbb{N}, \lambda \in(0, \infty), f \in \mathcal{S}^{\prime}\left(\mathbb{R}^{n}\right)$ and $\psi \in \mathcal{S}\left(\mathbb{R}^{n}\right)$ with $\widehat{\psi}(0)=0$. By using the definition of $S_{k_{0}}(f)$ and exchanging the order of integrals, we see that 


$$
\begin{aligned}
& b^{k_{0}(1-q)} \int_{U^{\complement}}\left[S_{k_{0}}(f)(x)\right]^{2} \varphi(x, \lambda) d x \\
= & b^{-q k_{0}} \sum_{k \in \mathbb{Z}} b^{-k} \int_{U^{\complement}}\left|f * \psi_{-k}(y)\right|^{2} \varphi\left(\left(y+B_{k_{0}+k}\right) \cap U^{\complement}, \lambda\right) d y .
\end{aligned}
$$

Moreover, for any $y \in U^{\complement}$, by Lemma 2.15 with $\varphi \in \mathbb{A}_{q}(A)$ and Lemma 3.11(ii), we conclude that

$$
\begin{aligned}
\varphi\left(y+B_{k_{0}+k}, \lambda\right) & \lesssim b^{q k_{0}} \varphi\left(y+B_{k}, \lambda\right) \lesssim b^{q k_{0}} \frac{\left|y+B_{k}\right|^{q}}{\left|\left(y+B_{k}\right) \cap E^{\complement}\right|^{q}} \varphi\left(\left(y+B_{k}\right) \cap E^{\complement}, \lambda\right) \\
& \lesssim b^{q k_{0}} \varphi\left(\left(y+B_{k}\right) \cap E^{\complement}, \lambda\right) .
\end{aligned}
$$

From this and Lemma 2.15, it follows that the last integral in (3.12) is not more than a positive constant multiple of

$$
\sum_{k} b^{-k} \int_{U^{\complement}}\left|f * \psi_{-k}(y)\right|^{2} \varphi\left(\left(y+B_{k}\right) \cap E^{\complement}, \lambda\right) d y \lesssim \int_{E^{\complement}}[S(f)(x)]^{2} \varphi(x, \lambda) d x .
$$

This finishes the proof of Lemma 3.12.

To obtain the $g_{\lambda}^{*}$-function characterization of $H_{A}^{\varphi}\left(\mathbb{R}^{n}\right)$, the following technical lemma plays a key role, whose proof is motivated by Folland and Stein [17, p. 218, Theorem 7.1], Aguilera and Segovia [1, Theorem 1] and Liang et al. [34, Lemma 4.6].

Lemma 3.13. Let $q \in[1, \infty), \varphi \in \mathbb{A}_{q}(A)$ be an anisotropic growth function. Then there exists a positive constant $C$ such that, for all $k_{0} \in \mathbb{N}$ and measurable functions $f$,

$$
\int_{\mathbb{R}^{n}} \varphi\left(x, S_{k_{0}}(f)(x)\right) d x \leq C b^{\left(q-\frac{p}{2}\right) k_{0}} \int_{\mathbb{R}^{n}} \varphi(x, S(f)(x)) d x .
$$

Proof. For all $\lambda \in(0, \infty)$ and $k_{0} \in \mathbb{Z}_{+}$, let

$$
E_{\lambda, k_{0}}:=\left\{x \in \mathbb{R}^{n}: S(f)(x)>\lambda b^{k_{0} / 2}\right\}
$$

and

$$
U_{\lambda, k_{0}}:=\left\{x \in \mathbb{R}^{n}: \mathcal{M}_{A}\left(\chi_{E_{\lambda, k_{0}}}\right)(x)>b^{-k_{0}-2 \sigma}\right\},
$$

where $\mathcal{M}_{A}$ is as in (2.4). Then, by the weighted week type $(q, q)$ of $\mathcal{M}_{A}$ with $q \in[1, \infty)$ and $\varphi \in \mathbb{A}_{q}(A)$ (see, for example, [7, Theorem 2.4]), we have

$$
\varphi\left(U_{\lambda, k_{0}}, \lambda\right) \lesssim b^{q k_{0}}\left\|\chi_{E_{\lambda, k_{0}}}\right\|_{L^{q}\left(\mathbb{R}^{n}, \varphi(\cdot, \lambda)\right)} \sim b^{q k_{0}} \varphi\left(E_{\lambda, k_{0}}, \lambda\right) .
$$

From this and Lemma 3.12 with $E:=E_{\lambda, k_{0}}$ and $U:=U_{\lambda, k_{0}}$, it follows that 


$$
\begin{aligned}
& \varphi\left(\left\{x \in \mathbb{R}^{n}: S_{k_{0}}(f)(x)>\lambda\right\}, \lambda\right) \\
\leq & \varphi\left(U_{\lambda, k_{0}}, \lambda\right)+\varphi\left(\left(U_{\lambda, k_{0}}\right)^{\complement} \cap\left\{x \in \mathbb{R}^{n}: S_{k_{0}}(f)(x)>\lambda\right\}, \lambda\right) \\
\lesssim & b^{q k_{0}} \varphi\left(E_{\lambda, k_{0}}, \lambda\right)+\lambda^{-2} \int_{\left(U_{\lambda, k_{0}}\right)^{\complement}}\left[S_{k_{0}}(f)(x)\right]^{2} \varphi(x, \lambda) d x \\
\lesssim & b^{q k_{0}} \varphi\left(E_{\lambda, k_{0}}, \lambda\right)+b^{(q-1) k_{0}} \lambda^{-2} \int_{\left(E_{\lambda, k_{0}}\right)^{\complement}}[S(f)(x)]^{2} \varphi(x, \lambda) d x \\
\sim & b^{q k_{0}} \varphi\left(E_{\lambda, k_{0}}, \lambda\right)+b^{(q-1) k_{0}} \lambda^{-2} \int_{0}^{\lambda b^{k_{0} / 2}} t \varphi\left(\left\{x \in \mathbb{R}^{n}: S(f)(x)>t\right\}, \lambda\right) d t .
\end{aligned}
$$

By this, the fact that $\widetilde{\varphi} \sim \varphi$, where $\widetilde{\varphi}(x, t):=\int_{0}^{t} \varphi(x, s) d s / s$ for all $(x, t) \in \mathbb{R}^{n} \times$ $[0, \infty)$ (see [30, Lemma 4.1(ii)]), $k_{0} \in \mathbb{N}$ and the uniformly lower type $p$ and the uniformly upper type 1 properties of $\varphi$, we further conclude that

$$
\begin{aligned}
& \int_{\mathbb{R}^{n}} \varphi\left(x, S_{k_{0}}(f)(x)\right) d x \\
\sim & \int_{0}^{\infty} \frac{1}{\lambda} \varphi\left(\left\{x \in \mathbb{R}^{n}: S_{k_{0}}(f)(x)>\lambda\right\}, \lambda\right) d \lambda \\
\lesssim & b^{q k_{0}} \int_{0}^{\infty} \frac{1}{\lambda} \varphi\left(E_{\lambda, k_{0}}, \lambda\right) d \lambda \\
& +b^{(q-1) k_{0}} \int_{0}^{\infty} \lambda^{-3} \int_{0}^{\lambda b^{k_{0} / 2}} t \varphi\left(\left\{x \in \mathbb{R}^{n}: S(f)(x)>t\right\}, \lambda\right) d t d \lambda \\
\lesssim & b^{(q-p / 2) k_{0}} \int_{0}^{\infty} \frac{1}{\lambda} \varphi\left(\left\{x \in \mathbb{R}^{n}: S(f)(x)>\lambda\right\}, \lambda\right) d \lambda \\
& +b^{(q-1) k_{0}}\left[\int_{0}^{\infty} \lambda^{-3} \int_{0}^{\lambda} \lambda \varphi\left(\left\{x \in \mathbb{R}^{n}: S(f)(x)>t\right\}, t\right) d t d \lambda\right. \\
& \left.+\int_{0}^{\infty} \lambda^{-3} \int_{\lambda}^{\lambda b^{k_{0} / 2}}\left(\frac{\lambda}{t}\right)^{p} t \varphi\left(\left\{x \in \mathbb{R}^{n}: S(f)(x)>t\right\}, t\right) d t d \lambda\right] \\
\lesssim & b^{(q-p / 2) k_{0}} \int_{\mathbb{R}^{n}} \varphi(x, S(f)(x)) d x+b^{(q-1) k_{0}}\left\{\int_{0}^{\infty} \frac{1}{t} \varphi\left(\left\{x \in \mathbb{R}^{n}: S(f)(x)>t\right\}, t\right) d t\right. \\
& \left.+\int_{0}^{\infty} \frac{1}{t}\left[b^{(2-p) k_{0} / 2}-1\right] \varphi\left(\left\{x \in \mathbb{R}^{n}: S(f)(x)>t\right\}, t\right) d t\right\} \\
\lesssim & b^{(q-p / 2) k_{0}} \int_{\mathbb{R}^{n}} \varphi(x, S(f)(x)) d x .
\end{aligned}
$$

This finishes the proof of Lemma 3.13.

Proof of Theorem 3.9. The proof of Theorem 3.9 is now similar to that of [34, Proposition 4.7]. For all $f \in \mathcal{S}^{\prime}\left(\mathbb{R}^{n}\right)$ and $x \in \mathbb{R}^{n}$, since $S(f)(x) \leq g_{\lambda}^{*}(f)(x)$, the 
inequality $\|S(f)\|_{L^{\varphi}\left(\mathbb{R}^{n}\right)} \leq\left\|g_{\lambda}^{*}(f)\right\|_{L^{\varphi}\left(\mathbb{R}^{n}\right)}$ is obvious.

Conversely, for all $f \in \mathcal{S}^{\prime}\left(\mathbb{R}^{n}\right)$ and $x \in \mathbb{R}^{n}$, we have

$$
\begin{aligned}
& {\left[g_{\lambda}^{*}(f)(x)\right]^{2} } \\
= & \sum_{k \in \mathbb{Z}} b^{-k} \int_{x+B_{k}}\left|f * \varphi_{-k}(y)\right|^{2}\left[\frac{b^{k}}{b^{k}+\rho(x-y)}\right]^{\lambda} d y \\
& +\sum_{j=1}^{\infty} \sum_{k \in \mathbb{Z}} \int_{x+\left(B_{k+j} \backslash B_{k+j-1}\right)} \cdots \lesssim[S(f)(x)]^{2}+\sum_{j=1}^{\infty} b^{-j(\lambda-1)}\left[S_{j}(f)(x)\right]^{2} .
\end{aligned}
$$

By this, Lemmas 3.10 and 3.13 and $\lambda \in(2 q / p, \infty)$, we conclude that

$$
\begin{aligned}
& \int_{\mathbb{R}^{n}} \varphi\left(x, g_{\lambda}^{*}(f)(x)\right) d x \\
\lesssim & \sum_{j=0}^{\infty} \int_{\mathbb{R}^{n}} \varphi\left(x, b^{-j(\lambda-1) / 2} S_{j}(f)(x)\right) d x \\
\lesssim & \sum_{j=0}^{\infty} b^{-j p(\lambda-1) / 2} b^{j(q-p / 2)} \int_{\mathbb{R}^{n}} \varphi(x, S(f)(x)) d x \\
\lesssim & \int_{\mathbb{R}^{n}} \varphi(x, S(f)(x)) d x,
\end{aligned}
$$

which implies that $\left\|g_{\lambda}^{*}(f)\right\|_{L^{\varphi}\left(\mathbb{R}^{n}\right)} \lesssim\|S(f)\|_{L^{\varphi}\left(\mathbb{R}^{n}\right)}$. This finishes the proof of (3.10) and hence, together with Theorem 2.8, the proof of Theorem 3.9.

\section{ACKNOWLEDGMENTS}

The authors would like to thank the referees for their very careful reading and helpful comments which improve the presentation of this article.

\section{REFERENCES}

1. N. Aguilera and C. Segovia, Weighted norm inequalities relating the $g_{\lambda}^{*}$ and the area functions, Studia Math., 61 (1977), 293-303.

2. K. Astala, T. Iwaniec, P. Koskela and G. Martin, Mappings of BMO-bounded distortion, Math. Ann., 317 (2000), 703-726.

3. Z. Birnbaum and W. Orlicz, Über die verallgemeinerung des begriffes der zueinander konjugierten potenzen, Studia Math., 3 (1931), 1-67.

4. M. Bownik, Anisotropic Hardy spaces and wavelets, Mem. Amer. Math. Soc., 164 (2003), no. 781, vi+122 pp. 
5. M. Bownik, Boundedness of operators on Hardy spaces via atomic decompositions, Proc. Amer. Math. Soc., 133 (2005), 3535-3542.

6. M. Bownik, Anisotropic Triebel-Lizorkin spaces with doubling measures, J. Geom. Anal., 17 (2007), 387-424.

7. M. Bownik and K.-P. Ho, Atomic and molecular decompositions of anisotropic TriebelLizorkin spaces, Trans. Amer. Math. Soc., 358 (2006), 1469-1510.

8. M. Bownik, B. Li, D. Yang and Y. Zhou, Weighted anisotropic Hardy spaces and their applications in boundedness of sublinear operators, Indiana Univ. Math. J., 57 (2008), 3065-3100.

9. M. Bownik, B. Li, D. Yang and Y. Zhou, Weighted anisotropic product Hardy spaces and boundedness of sublinear operators, Math. Nachr., 283 (2010), 392-442.

10. H. Q. Bui, Weighted Hardy spaces, Math. Nachr., 103 (1981), 45-62.

11. M. Christ, A $T(b)$ theorem with remarks on analytic capacity and the Cauchy integral, Colloq. Math., 60/61 (1990), 601-628.

12. D.-C. Chang, D. Yang and S. Yang, Real-variable theory of Orlicz-type function spaces associated with operators - a survey, Submitted, 2013.

13. S.-Y. A. Chang and R. Fefferman, A continuous version of duality of $H^{1}$ with BMO on the bidisc, Ann. of Math. (2), 112 (1980), 179-201.

14. R. R. Coifman and G. Weiss, Analyse Harmonique Non-commutative sur Certains Espaces Homogènes, Lecture Notes in Math., 242, Springer, Berlin, 1971.

15. L. Diening, Maximal function on Musielak-Orlicz spaces and generalized Lebesgue spaces, Bull. Sci. Math., 129 (2005), 657-700.

16. C. Fefferman and E. M. Stein, $H^{p}$ spaces of several variables, Acta Math., 129 (1972), 137-193.

17. G. B. Folland and E. M. Stein, Hardy Spaces on Homogeneous Groups, Mathematical Notes, 28, Princeton University Press, Princeton, N. J.; University of Tokyo Press, Tokyo, 1982, xii +285 pp.

18. M. Frazier, B. Jawerth and G. Weiss, Littlewood-Paley Theory and the Study of Function Spaces, CBMS Regional Conference Series in Mathematics, 79, Published for the Conference Board of the Mathematical Sciences, Washington, DC; by the American Mathematical Society, Providence, RI, 1991.

19. J. García-Cuerva, Weighted $H^{p}$ spaces, Dissertationes Math. (Rozprawy Mat.), 162 (1979), 1-63.

20. J. García-Cuerva and J. M. Martell, Wavelet characterization of weighted spaces, J. Geom. Anal., 11 (2001), 241-264.

21. J. García-Cuerva and J. L. Rubio de Francia, Weighted Norm Inequalities and Related Topics, North-Holland, Amsterdam, 1985. 
22. L. Grafakos, Classical Fourier Analysis, Second edition, Graduate Texts in Mathematics, 249, Springer, New York, 2008.

23. L. Grafakos, Modern Fourier Analysis, Second edition, Graduate Texts in Mathematics, 250, Springer, New York, 2009.

24. S. Hou, D. Yang and S. Yang, Lusin area function and molecular characterizations of Musielak-Orlicz Hardy spaces and their applications, Commun. Contemp. Math., 15(6) (2013), 1350029, 37 pp.

25. T. Iwaniec and J. Onninen, $\mathcal{H}^{1}$-estimates of Jacobians by subdeterminants, Math. Ann., 324 (2002), 341-358.

26. S. Janson, On functions with conditions on the mean oscillation, Ark. Mat., 14 (1976), 189-196.

27. S. Janson, Generalizations of Lipschitz spaces and an application to Hardy spaces and bounded mean oscillation, Duke Math. J., 47 (1980), 959-982.

28. R. Jiang and D. Yang, New Orlicz-Hardy spaces associated with divergence form elliptic operators, J. Funct. Anal., 258 (2010), 1167-1224.

29. T. Kilpeläinen, P. Koskela and H. Masaoka, Harmonic Hardy-Orlicz spaces, Ann. Acad. Sci. Fenn. Math., 38 (2013), 309-325.

30. L. D. Ky, New Hardy spaces of Musielak-Orlicz type and boundedness of sublinear operators, Integral Equations Operator Theory, 78 (2014), 115-150.

31. L. D. Ky, Bilinear decompositions and commutators of singular integral operators, Trans. Amer. Math. Soc., 365 (2013), 2931-2958.

32. L. D. Ky, Bilinear decompositions for the product space $H_{L}^{1} \times B M O_{L}$, Math. Nachr., (2013), DOI: 10.1002/mana201200101 (to appear) or arXiv: 1204.3041.

33. B. Li, D. Yang and W. Yuan, Anisotropic Hardy spaces of Musielak-Orlicz type with applications to boundedness of sublinear operators, The Scientific World Journal, Vol. 2014, Article ID 306214, 19 pages, 2014. doi: 10.1155/2014/306214.

34. Y. Liang, J. Huang and D. Yang, New real-variable characterizations of Musielak-Orlicz Hardy spaces, J. Math. Anal. Appl., 395 (2012), 413-428.

35. Y. Liang and D. Yang, Musielak-Orlicz Campanato spaces and applications, J. Math. Anal. Appl., 406 (2013), 307-322.

36. S. Martínez and N. Wolanski, A minimum problem with free boundary in Orlicz spaces, Adv. Math., 218 (2008), 1914-1971.

37. B. Muckenhoupt and R. Wheeden, On the dual of weighted $H^{1}$ of the half-space, Studia Math., 63 (1978), 57-79.

38. J. Musielak, Orlicz Spaces and Modular Spaces, Lecture Notes in Math., 1034, SpringerVerlag, Berlin, 1983.

39. E. Nakai and Y. Sawano, Orlicz-Hardy spaces and their duals, Sci. China Math., 57 (2014), 903-962. 
40. W. Orlicz, Über eine gewisse Klasse von Räumen vom Typus B, Bull. Int. Acad. Pol. Ser. A, 8 (1932), 207-220.

41. E. M. Stein and G. Weiss, Introduction to Fourier Analysis on Euclidean Spaces, Princeton Mathematical Series, No. 32, Princeton University Press, Princeton, N. J., 1971.

42. J.-O. Strömberg, Bounded mean oscillation with Orlicz norms and duality of Hardy spaces, Indiana Univ. Math. J., 28 (1979), 511-544.

43. J.-O. Strömberg and A. Torchinsky, Weighted Hardy Spaces, Lecture Notes in Mathematics, 1381, Springer-Verlag, Berlin/New York, 1989.

44. H. Triebel, Theory of Function Spaces, Birkhäuser, Basel, 1983.

Baode Li and Xingya Fan

School of Mathematics and System Sciences

Xinjiang University

Urumqi 830046

People's Republic of China

E-mail: baodeli@xju.edu.cn 1287522641@qq.com

Dachun Yang

School of Mathematical Sciences

Beijing Normal University

Laboratory of Mathematics and Complex Systems

Ministry of Education

Beijing 100875

People's Republic of China

E-mail: dcyang@bnu.edu.cn 